\title{
La productividad multifactorial: concepto, medición y significado ${ }^{1}$
}

\author{
Enrique Hernández Laos ${ }^{2}$
}

\section{RESUMEN}

En la bibliografía empírica del crecimiento económico suele hacerse referencia a las llamadas "fuentes del crecimiento." Ello implica la medición y conceptualización de la llamada productividad multifactorial, lo que no constituye una tarea sencilla. En este artículo se exponen algunos de los principales problemas para la medición de la productividad multifactorial, y se avanza en la discusión de su conceptualización con fines de análisis empírico del crecimiento de los países. Por último, se ofrece un método para su medición basado en números índices que busca evitar las principales críticas teóricas que se hacen a los enfoques más comunes a que están sujetas este tipo de mediciones.

\section{Abstract}

It is usual in empirical literature of economic growth to make reference to the "sources of growth." That implies the conceptualization and measurement of the so-called multifactor productivity, which by no means constitute an obvious task. In this article, the various problems involved in its measurement are exposed, and it is advanced some implications for its conceptualization. Finally, the author proposes an alternative method for its measurement based on an index-number approach which tries to avoid some of the main critical objections to other approaches present in the specialized literature.

Número de clasificación: JEL: C10, O30, O40.

Palabras clave: Crecimiento económico, productividad multifactorial, cambio tecnológico.

\footnotetext{
${ }^{1}$ Adaptación del capítulo III del libro de Enrique Hernández Laos: La productividad y el desarrollo económico de México (1950-2000) (En proceso.) Centro de Estudios Latinoamericanos, Universidad de Stanford, CA., febrero de 2007.

${ }^{2}$ Profesor-investigador. Universidad Autónoma Metropolitana, Unidad Iztapalapa, ehlaos@ prodigy.net.mx.
} 


\section{INTRODUCCIÓN}

En un sentido intuitivo suele considerarse a la productividad laboral como una medida de la eficiencia con que se aprovechan los recursos humanos de un país. Y tal consideración, si bien tiene una dosis de verdad, no es totalmente acertada porque, como mucho se ha hecho notar por parte de los economistas, aumentos significativos del producto por hombre ocupado pueden estar reflejando no solamente una mejor utilización de los esfuerzos laborales del país, sino también pueden ser consecuencia de un proceso de sustitución factorial. Esto es, podría ser que crecientes dotaciones de maquinaria y equipo estén sustituyendo con esfuerzo mecánico y automático una parte creciente de lo que anteriormente se realizaba por medio de la aplicación de esfuerzo humano. En este caso, se dice, se estaría presentando una "profundización de capital" por hombre ocupado, uno de cuyos resultados sería una mayor capacidad de generar producto por hombre activo u ocupado en la economía (Kendrik, 1961; Hernández Laos, 1973; Diewert y Lawrence, 1999).

Debe tenerse en cuenta, sin embargo, que la utilización de procesos productivos más intensivos en capital es posible alcanzarlos sólo con inversiones en diversos tipos de activos fijos. A escala agregada de un país, ello sólo es realizable a través de un proceso de acumulación de capital, que por su propia naturaleza resulta oneroso para las unidades productivas. Por ello, debería ser posible considerar tanto la magnitud del esfuerzo humano, como su posible sustitución por fuerza mecánica o automatizada debida a la creciente utilización de activos fijos y otros bienes de capital, dentro de una medida conjunta de la productividad. Esto último con el objeto de separar, por una parte, la fracción de los aumentos del producto que derivan de una mayor utilización de recursos (que implican un costo para la sociedad), de otra fracción que -en teoría- deriva de una mejor eficiencia en la utilización de los recursos y que, en principio, no es onerosa para la sociedad en su conjunto.

No estamos diciendo nada nuevo. Griliches (1996), en una muy breve nota histórica, relata cómo el intento de relacionar el producto, no sólo con la mano de obra, sino con una miríada de otros recursos (capital, tierra) en el intento de medir la productividad de las naciones data, en la bibliografía especializada, cuando menos desde los años treinta del siglo pasado. ${ }^{3}$ El punto de partida de

\footnotetext{
${ }^{3}$ Según Griliches (1996), el primero en discutir una medida estadística de esta naturaleza habría sido Morris Copeland (1937), y una aproximación más clara la encontraríamos en Copeland y Martin (1938). Desde una perspectiva teórica, el concepto había sido discutido de manera explícita por John Hicks desde principios de los cuarenta (Hicks, 1940).
} 
este enfoque lo aporta, por supuesto, el sistema de cuentas nacionales. En ese enfoque, los mercados de productos y de factores se conectan por la igualación de los ingresos y los gastos en un año base de comparación. De esta manera, en los años subsiguientes, si la identidad se desagrega en su componente de precios y cantidades, tanto de productos como de factores, dicha identidad se pierde si se registran cambios en la eficiencia con que se usan los recursos, porque una mayor cantidad de producto puede generarse con una cuantía dada de insumos, sólo si se introduce un escalar que restituya la igualdad. Desde ese punto de vista, en el año base el escalar es igual a la unidad, y en los años siguientes éste puede ser igual, mayor o menor que la unidad, lo que indica la constancia, el aumento o la disminución de la eficiencia y productividad conjunta de los factores productivos (Hulten, 2001).

Durante varias décadas, el desarrollo empírico del concepto de la productividad multifactorial, anteriormente bosquejado, avanzó de manera gradual pero sistemática ${ }^{4}$ No sería, sin embargo, hasta la contribución seminal de Solow (1957) que se tendría de manera relativamente integrada, tanto teórica como empíricamente, el concepto de la productividad multifactorial, la cual en su momento se identificó (equivocadamente como veremos más adelante) con el cambio tecnológico. Solow (1957) identifica, así, el "cambio tecnológico" con los desplazamientos neutrales de la función de producción, pero sin necesidad de cuantificarla directamente, dados algunos supuestos específicos sobre la naturaleza de la tecnología (rendimientos constantes a escala) y sobre el comportamiento de los productores (mercados competitivos de productos y factores). Si el desplazamiento de la función de producción es neutral, al sucederse tales desplazamientos no se alteran las tasas marginales de sustitución, por lo que el factor multiplicativo representa el efecto acumulativo de los desplazamientos de la función de producción a lo largo del tiempo.

Dadas esas premisas teóricas, el desplazamiento anual de la función de producción puede medirse como la diferencia entre la tasa de crecimiento del producto y la tasa (combinada) de crecimiento de los insumos de factores, ponderadas cada una de ellas por su respectiva participación en el ingreso. Si este

\footnotetext{
${ }^{4}$ Griliches (1996) destaca, por ejemplo, los trabajos estadísticos en esta dirección desarrollados por Tinbergen (1942),Tintner (1944), Stigler, 1947; Barton y Cooper, 1948; Johnson (1950), Schmookler (1952), Fabricant (1954); Kendrick (1955) y Abramovitz (1956).

${ }^{5}$ Vale la pena hacer mención que el concepto de "cambio tecnológico" adelantado por Solow era de carácter más bien laxo, y es de creerse que ni siquiera ponía el énfasis en los avances de la tecnología, como lo demuestra la siguiente observación: "Utilizo la frase 'cambio tecnológico' como una expresión corta que defina cualquier clase de desplazamiento en la función de producción” (Solow, 1957: 312; énfasis nuestro).
} 
esquema es aceptado -argumenta Solow- todo lo que se necesita para medir los cambios (anuales y acumulados) en la productividad multifactorial son las series de producto por hora-hombre, las de capital por hora-hombre y la participación de los ingresos de capital en el valor del producto bruto nacional. Esta es la base de lo que posteriormente se conocería como la contabilidad del crecimiento, cuya aplicación empírica permite identificar de manera separada las contribuciones del crecimiento de los recursos (capital por hora-hombre empleada) y la del cambio tecnológico (productividad multifactorial) al crecimiento de la productividad del trabajo (producto por hora-hombre trabajada). Este esquema contable ha sido considerado, desde hace ya varias décadas, como un paso preliminar para el análisis de los determinantes fundamentales del crecimiento económico, en la medida en que los factores que determinan la acumulación de recursos difieren de los que inciden -y explican- el acrecentamiento de la productividad multifactorial (Barro, 1999; Barro y Sala-i-Martin, 2005).

En el enfoque descrito, la productividad multifactorial se calcula empíricamente como un residuo, y así es precisamente como suele denominársele: "el residuo de Solow". El problema, sin embargo, es que en la práctica no sabemos qué parte de ese residuo es sistemática y qué parte obedece a sesgos de medición. Es decir, qué parte de la productividad multifactorial así estimada refleja un comportamiento sustantivo de modificaciones en la productividad y en la eficiencia con que se utilizan los factores productivos, y qué parte obedece simplemente a errores de medición que se acumulan en el cálculo residual de la misma. Por ello es que los problemas de medición adoptan un papel muy relevante en las discusiones -tanto teóricas como aplicadas- de la productividad multifactorial en el contexto de la teoría del crecimiento.

Por ejemplo, Hulten (2001) señala que son tres los sesgos más usuales que tienen implicaciones en la medición de la productividad:

- El que deriva de la no observancia del supuesto de la existencia de rendimientos constantes a escala (Teorema de Euler), que permite que la suma de las participaciones factoriales en el ingreso se ajuste a la unidad.

- $\quad$ El que resulta de utilizar las participaciones relativas de los factores en el producto, lo que reclama del supuesto de que los factores se pagan de acuerdo a su productividad marginal,

- Y el supuesto de que el cambio tecnológico deba ser hicks-neutral, es decir, que el cambio tecnológico no afecta de manera diferente las productividades marginales de cada uno de los factores de producción. 
Resulta claro, sin embargo, que los anteriores constituyen sesgos de medición sólo en tanto se insista en equiparar los cambios de la productividad multifactorial con el cambio tecnológico concebido de manera restringida, esto es, como resultado de desplazamientos de la función de producción que derivan únicamente de la introducción de nuevas tecnologías. Si esa equiparación no se realiza y los movimientos en la productividad multifactorial se consideran más bien -como más adelante veremos- como cambios en la eficiencia con la que se utilizan los factores productivos, el no cumplimiento de los supuestos mencionados no imprime, de hecho, ningún sesgo significativo en la medición de la productividad multifactorial, sino que modifica el significado de lo que tales mediciones implican en términos de eficiencia. ${ }^{6}$ Así, los problemas de medición de la productividad, cuando se hace a un lado la pretensión de medir el cambio tecnológico, modifican el significado de lo que "realmente" se está midiendo, sobre todo cuando los innumerables supuestos neoclásicos descritos no se presentan en la realidad.

Pero los problemas de medición van más allá de la materialización o no de tales supuestos. Desde el punto de vista de la medición, por ejemplo, las discusiones van desde la pretensión de la desaparición de la productividad si los insumos están "adecuadamente" medidos (Jorgenson y Griliches, 1967), a la discusión de los problemas involucrados en la agregación de insumos y de productos (OCDE, 2001); y desde la afirmación de la equivalencia que resulta de calcular la PTF como un residuo y calcularla como un cociente de números índices de productos a insumos y, por lo tanto, la pertinencia de discutir las diferentes formulaciones de los números índices aplicables para la medición de la productividad (Diewert, 1982; Diewert, 1999; Diewert y Nakamura, 2003), hasta la controversia sobre la necesidad de considerar de manera separada los cambios tecnológicos y los

${ }_{6}$ Por ejemplo, para Hulten (2001) no hay nada que obligue a hacer el primer supuesto en la medición de la productividad multifactorial, si las participaciones de los factores se calculan de manera no residual. El supuesto de la existencia de mercados competitivos, por otra parte, es común que no se cumpla en la realidad, por lo que en presencia de mercados imperfectos en los cuales el precio es mayor que el costo marginal, o en presencia de rendimientos crecientes a escala, las mediciones de la productividad multifactorial dejan de constituir solo desplazamientos neutrales de la función de producción, para convertirse en una expresión combinada de este y de los rendimientos crecientes a escala, como lo demuestra Hall (1988). Por último, si el componente de cambio tecnológico implícito en la productividad multifactorial no es estrictamente neutral, sus movimientos pueden resultar no solo de desplazamientos de la función de producción, sino también de modificaciones en las participaciones relativas de los factores (Hernández Laos, 1985). Ello implica, como mas adelante veremos, que la evolución de la productividad deja de ser sendero-independiente, es decir, la medición de la productividad resulta influida por la secuencia de las mediciones anuales. 
cambios en la eficiencia de las empresas en la medición de la productividad multifactorial (Färe et al., 1994b; Coelli et al., 1998). Los desarrollos más recientes ponen el énfasis en la medición de la productividad a nivel de planta (Bartelsman y Doms, 2000; Tybout, 2000; Baily y Solow 2001; Foster, L., J. Haltinwagner y C. J. Krizan, 2001) en la endogenización de los gastos de I y D (Griliches, 1994), y en la medición de la productividad a través de precios relativos de insumos y productos en el llamado "enfoque dual" (Hsieh, 1998).

Vale apuntar, por último, que las nuevas teorías del crecimiento endógeno han traído a la mesa de discusión problemas de medición e interpretación de la productividad, esto es, el significado que cobran los cambios en la misma y su papel en el crecimiento económico, por medio de desbordamientos (spillovers), de la introducción de nuevos productos o cambios en la calidad de los mismos, y de la inclusión -o no- de los llamados activos intangibles en la medición e interpretación de la productividad multifactorial.

En la primera sección se aborda brevemente el significado de los conceptos de eficiencia y productividad, y en la segunda se describen de manera sucinta los números índices que más suelen utilizarse en la medición de la productividad multifactorial. El tercer apartado describe otros enfoques para medir la productividad, citados en la bibliografía especializada, y se evalúa su pertinencia. El cuarto apartado describe los métodos de cuantificación de la productividad que proponemos sean utilizados en investigaciones empíricas. El quinto inciso intenta ofrecer una reflexión sobre el significado de las mediciones de productividad que estamos proponiendo, en tanto que en el anexo se ofrece la derivación matemática de nuestro enfoque para la medición de la productividad multifactorial.

\section{Conceptos de Eficiencia y Productividad}

En el lenguaje común, productividad suele ser sinónimo de eficiencia. De hecho, el concepto de eficiencia deriva de su aplicación en términos ingenieriles, según los cuales un proceso (o una máquina) es "eficiente" si, dado un conjunto de insumos, el proceso (la máquina) es capaz de generar la máxima producción posible, que suele estar determinada por la máxima capacidad alcanzable por unidad de tiempo (Roscoe, 1963).

En una contribución destacada presentada hace ya varias décadas, un economista analizó con mayor detenimiento el concepto de eficiencia, desde un punto de vista de la teoría de la producción (Farell, 1957). Farell examinó la estructura de la eficiencia productiva de las empresas, la cual puede ser considerada 
como el producto de dos términos: la eficiencia técnica y la eficiencia asignativa (o eficiencia precio); propuso una forma de cuantificar ambos tipos de eficiencia y, lo que es más, ofreció una interpretación de ambas en términos de costos. La eficiencia técnica, en el sentido dado por Farell, tiene mucho en común con su consideración meramente ingenieril, esto es, dada una superficie de producción para la empresa, delimitada por una función de producción con determinadas características, la eficiencia técnica se logra si la empresa opera en cualesquiera puntos de esa frontera de producción, es decir, si dada la utilización de insumos, la empresa es capaz de generar la máxima producción que es técnicamente posible, dada la tecnología empleada. Si por cualquier razón ello no es posible, se dice que la empresa opera de manera técnicamente ineficiente. ${ }^{7}$

El otro tipo de eficiencia apuntado por Farell se refiere a la asignación de los recursos. En este caso intervienen los precios en la evaluación: si una empresa enfrenta un producto constante y unos precios (dados) de los insumos que utiliza, se dice que esos insumos están eficientemente asignados si los productos generados y los insumos utilizados minimizan los costos con los que opera la empresa en la producción de ese volumen específico de producto. Es claro que la eficiencia en la asignación de los recursos implica la eficiencia técnica de la empresa porque, para minimizar los costos, la combinación de insumos elegida por la empresa debe estar en la frontera de la superficie de producción (en la curva de isocuanta por ejemplo). Sin embargo, al alcanzar la eficiencia técnica la empresa no necesariamente logra la eficiencia asignativa, porque los productos y los insumos pueden estar en la frontera de producción pero no minimizar los costos de la unidad productiva. ${ }^{8}$

\footnotetext{
7 En términos sencillos, la empresa es técnicamente eficiente si opera, por ejemplo, a lo largo de una isocuanta que describe las combinaciones posibles de (dos) insumos para generar un producto dado alcanzable. Para la economía neoclásica de la producción (Carlson, 1939; Hicks, 1946; Samuelson, 1947) todas las empresas operan, por definición, de manera técnicamente eficiente, lo que no siempre sucede en la realidad, como lo vino a mostrar la aplicación de técnicas de programación lineal a los problemas de producción de las empresas. Otros desarrollos teóricos posteriores vinieron a confirmar que la presencia de ineficiencias técnicas podría ser más bien la regla que la excepción (Salter, 1966: Leibenstein, 1966). Para una discusión general véase: Hernández Laos (1985: Cap. 4); y para una discusión formal consúltese: Färe et al. (1985: Cap. 1) Los últimos autores ponen énfasis en el hecho de que la ineficiencia, aunque es privada, representa un costo social que debe ser considerado de manera explícita en la evaluación del desempeño de las economías.

8 Cuando el número de productos es mayor que uno y/o el volumen de producción es variable, la consideración anterior implica que la eficiencia asignativa sólo se alcanza si la empresa maximiza ganancias, es decir, no basta con la minimización de costos. Por supuesto, si la minimización de costos se logra para cada nivel de producción, ambas condiciones resultan equivalentes, si la empresa es tomadora de precios tanto en los mercados de factores como de productos.
} 
Parecería razonable que estuviésemos interesados en eliminar (o al menos disminuir) las ineficiencias técnicas de las empresas, las industrias o de la economía como un todo, porque de esa manera reduciríamos el desperdicio de recursos en la sociedad. Menos clara, sin embargo, es la insistencia de los economistas en eliminar (o reducir) las ineficiencias asignativas de las empresas. En este caso, desde los planteamientos originales (y relativamente confusos) de Pareto, hasta los más avanzados de Debreu (1951) y posteriores de Diewert (1983), sabemos que si no todas las empresas alcanzan la eficiencia asignativa, seria posible (al menos teóricamente) reasignar los recursos de manera que se produjera más de algún producto sin disminuir la producción de los demás, y sin necesidad de modificar la cantidad de recursos utilizados. Es decir, la falta de eficiencia asignativa en la empresa se traduce en ineficiencia técnica en la industria o de la economía como un todo, lo que obviamente implica un desperdicio de recursos o una pérdida potencial de producción dados esos mismos recursos. ${ }^{9}$

La discusión anterior considera, en todo caso, un concepto absoluto de eficiencia. Es sabido, sin embargo, que los análisis más usuales de eficiencia y productividad hacen referencia a un concepto relativo, es decir, comparativo de la misma. En efecto, suele tomarse como punto de referencia, por ejemplo, el de una empresa (o un conjunto de empresas) que se consideran apriorísticamente como un estándar alcanzable de eficiencia, en lo que se conoce como benchmarking de eficiencia o productividad. O puede tratarse, en todo caso, de comparaciones entre empresas o industrias de diversos países y, en el caso más frecuente, el análisis hace referencia a una empresa (industria o economía) en diferentes momentos en el tiempo, esto es, se toma como el estándar de referencia un año o periodo base de comparación, y se examina (se mide) la evolución de la eficiencia o productividad a lo largo del tiempo. En este último caso, se trata del análisis del cambio en las condiciones medias de eficiencia o productividad, y la manera más usual de cuantificarlo es a través de un cociente que relaciona la cuantía de los productos en dos momentos de, vis-à-vis de la cuantía de los insumos utilizados en su generación (Hernández Laos, 1985; Diewert, 1999).

\footnotetext{
${ }^{9}$ En este caso, sin embargo, es el sistema de precios el que debería asignar de manera adecuada los recursos, como lo muestra el llamado "coeficiente de utilización de recursos" discutido por Debreu (1951: 283), el cual mide, en forma escalar, el grado de ineficiencia del sistema económico en su totalidad, respecto de la situación -hipotética- que deriva de suponer que el sistema económico se encuentra en equilibrio óptimo de Pareto.
} 
En este punto, conviene señalar que el cambio de productividad así definido se acompaña de muy diferentes movimientos. En efecto, en los cambios en la productividad conceptualmente deberían intervenir tanto los aumentos en la eficiencia técnica de las empresas, es decir, los movimientos de éstas hacia su propia frontera de producción, así como muy posiblemente los cambios tecnológicos que estarían reflejando los desplazamientos de su frontera de producción. En teoría, los movimientos de la productividad así concebidos estarían reflejando de alguna manera los efectos de la existencia de rendimientos crecientes a escala, en otras palabras, movimientos a lo largo de la frontera de producción en respuesta al tamaño del mercado y a cambios en los precios relativos de los insumos. Por supuesto, si el análisis trasciende la unidad de producción y se realiza a escala de una industria, por ejemplo, o de un grupo de industrias, los movimientos de la productividad podrían obedecer, además, a cambios en la eficiencia-precio o eficiencia asignativa entre las empresas o las industrias, lo que se percibiría como efecto de aumentos de la eficiencia técnica en las industrias o en la economía nacional.

En suma, de lo discutido anteriormente se deriva que el concepto básico utilizado considera a la productividad como sinónimo de eficiencia, que se expresa como una relación entre productos e insumos. En un contexto inter temporal, hay varias formas de plantear esa relación entre productos e insumos. Para ejemplificar, y por razones de exposición, consideraremos primero el caso más sencillo, el de una empresa mono productora que sólo utiliza un insumo en su producción. Tenemos que el número de productos $(y)$ es uno $(\mathrm{n}=1)$ y el número de insumos $(x)$ es también uno $(\mathrm{m}=1) .^{10}$

Para una secuencia de periodos (anuales por ejemplo) especificados por $t=0$, $1, \ldots, T$, la cantidad de insumo utilizado en el periodo $t$ es $\mathrm{x}_{1 \mathrm{t}}$ cuyo precio unitario es $\mathrm{w}_{1 \mathrm{t}}$ y la cantidad de producto es $\mathrm{y}_{1 \mathrm{t}}$ cuyo precio es $\mathrm{p}_{1 \mathrm{t}}$. La definición básica de la productividad relaciona, como ya se hizo mención, los insumos con el producto a que dan lugar, y más específicamente, a través del cociente que resulta de dividir el producto por la cuantía del insumo, que es como suele especificarse en términos físicos $\left(\Pi_{t}\right)$ :

$$
\Pi_{\mathrm{t}} \equiv\left(\mathrm{y}_{1 \mathrm{t}} / \mathrm{x}_{1 \mathrm{t}}\right) \equiv \alpha_{\mathrm{t}}
$$

En donde $\alpha_{t}$ constituye un coeficiente de producto por unidad de insumo, que expresa una medición de la productividad $(\Pi)$ en el período " $t$ ". El coeficiente de producto-insumo $\left(\alpha_{t}\right)$ implica una relación entre un solo producto y un solo insumo, aunque más adelante veremos que da lugar a una expresión similar en el

${ }^{10}$ Los siguientes párrafos se basan en la presentación ofrecida por Diewert y Nakamura (2003). 
caso de una empresa fabricante de varios productos que utiliza varios insumos, siempre y cuando ambos (productos e insumos) estén agregados por medio de números índices.

Dado que estamos interesados en cuantificar los cambios en la productividad -y no los niveles en un período dado-, vamos a destacar las variaciones de la productividad en el tiempo, digamos entre el período "s" y el período "t", lo que puede lograrse a través de cuatro formulaciones distintas, las cuales enumeramos a continuación:

$$
\pi(1) \equiv\left(\mathrm{y}_{1 \mathrm{t}} / \mathrm{x}_{1 \mathrm{t}}\right) /\left(\mathrm{y}_{1 \mathrm{~s}} / \mathrm{x}_{1 \mathrm{~s}}\right)=\alpha_{\mathrm{t}} / \alpha_{\mathrm{s}}
$$

en donde $\pi$ (1) constituye una de las formulaciones, la cual se expresa como el cociente que resulta de dividir los coeficientes de producto por unidad de insumo, pertenecientes a los períodos " $\mathrm{t}$ " $\mathrm{y}$ " $\mathrm{s}$ " respectivamente.

Una segunda formulación del crecimiento de la productividad es considerarlo como el cociente que resulta de dividir el crecimiento del producto entre el crecimiento del insumo, que, en el caso simple que estamos analizando de un producto y un insumo, se expresa como:

$$
\pi(2) \equiv\left(\mathrm{y}_{1 \mathrm{t}} / \mathrm{y}_{1 \mathrm{~s}}\right) /\left(\mathrm{x}_{1 \mathrm{t}} / \mathrm{x}_{1 \mathrm{~s}}\right)
$$

La tercera y la cuarta formulaciones relacionan el crecimiento de la productividad $(\pi)$ con los ingresos y costos de la empresa, expresados estos en términos totales o en términos de márgenes de operación. En el caso de los ingresos totales $\left(\mathrm{R}_{\mathrm{t}}\right)$ y los costos totales $\left(\mathrm{C}_{1 \mathrm{t}}\right)$ de la empresa se tiene:

$$
\mathrm{R}_{\mathrm{t}} \equiv \mathrm{p}_{1 \mathrm{t}} \mathrm{y}_{1 \mathrm{t}} ; \mathrm{C}_{\mathrm{t}} \equiv \mathrm{w}_{1 \mathrm{t}} \mathrm{x}_{1 \mathrm{t}}
$$

Un poco de algebra elemental muestra que si se divide en cociente de los ingresos por el cociente de los precios de ambos períodos se tiene:

$$
\left(\mathrm{R}_{\mathrm{t}} / \mathrm{R}_{\mathrm{s}}\right) /\left(\mathrm{p}_{\mathrm{t}} / \mathrm{p}_{\mathrm{s}}\right)=\left(\mathrm{p}_{1 \mathrm{t}} \mathrm{y}_{1 \mathrm{t}} / \mathrm{p}_{1 \mathrm{~s}} \mathrm{y}_{1 \mathrm{~s}}\right) /\left(\mathrm{p}_{1 \mathrm{t}} / \mathrm{p}_{1 \mathrm{~s}}\right)=\mathrm{y}_{1 \mathrm{t}} / \mathrm{y}_{1 \mathrm{~s}}
$$

Y de manera similar, si se divide el cociente de los costos totales en los dos períodos por el cociente de los precios del insumo se obtiene:

$$
\left(\mathrm{C}_{\mathrm{t}} / \mathrm{C}_{\mathrm{s}}\right) /\left(\mathrm{w}_{\mathrm{t}} / \mathrm{w}_{\mathrm{s}}\right)=\left(\mathrm{w}_{1 \mathrm{t}} \mathrm{x}_{1 \mathrm{t}} / \mathrm{w}_{1 \mathrm{~s}} \mathrm{x}_{1 \mathrm{~s}}\right) /\left(\mathrm{w}_{1 \mathrm{t}} / \mathrm{w}_{1 \mathrm{~s}}\right)=\mathrm{x}_{1 \mathrm{t}} / \mathrm{x}_{1 \mathrm{~s}}
$$

Por lo que la tercera formulación de la productividad puede expresarse como el cociente del crecimiento de los ingresos entre el crecimiento de los costos, esto es:

$$
\pi(3) \equiv\left[\left(\mathrm{R}_{\mathrm{t}} / \mathrm{R}_{\mathrm{s}}\right) /\left(\mathrm{p}_{1 \mathrm{t}} / \mathrm{p}_{1 \mathrm{~s}}\right)\right] /\left[\left(\mathrm{C}_{\mathrm{t}} / \mathrm{C}_{\mathrm{s}}\right) /\left(\mathrm{w}_{1 \mathrm{t}} / \mathrm{w}_{1 \mathrm{~s}}\right)\right]=\left(\mathrm{y}_{1 \mathrm{t}} / \mathrm{y}_{1 \mathrm{~s}}\right) /\left(\mathrm{x}_{1 \mathrm{t}} / \mathrm{x}_{1 \mathrm{~s}}\right)
$$


Por último, y dado que las empresas se interesan en que los ingresos que perciben excedan los costos, el cálculo se realiza en términos de un margen de ganancia $\left(\mathrm{m}_{\mathrm{t}}\right)$ de manera que para el período téste margen puede ser medido como $\left(1+\mathrm{m}_{\mathrm{t}}\right) \equiv \mathrm{R}_{\mathrm{t}} / \mathrm{C}_{\mathrm{t}}$. Entonces la cuarta formulación de la productividad puede hacerse mediante la consideración explícita de los márgenes comerciales en ambos periodos, de la siguiente manera:

$$
\pi(4) \equiv\left[\left(1+\mathrm{m}_{\mathrm{t}}\right) /\left(1+\mathrm{m}_{\mathrm{s}}\right)\right]\left[\left(\mathrm{w}_{1 \mathrm{t}} / \mathrm{w}_{1 \mathrm{~s}}\right) /\left(\mathrm{p}_{1 \mathrm{t}} / \mathrm{p}_{1 \mathrm{~s}}\right)\right]=\left(\mathrm{y}_{1 \mathrm{t}} / \mathrm{y}_{1 \mathrm{~s}}\right) /\left(\mathrm{x}_{1 \mathrm{t}} / \mathrm{x}_{1 \mathrm{~s}}\right)
$$

Diewert y Nakamura (2003) interpretan el margen de ganancia como una recompensa al empresario por utilizar su capacidad administrativa como un insumo empresarial, por lo que $\pi$ (4) puede considerarse como el crecimiento en el precio de los insumos considerados de manera amplia (incluyendo la retribución a la capacidad empresarial) dividido por el crecimiento en el precio del producto generado. De nuevo, un poco de álgebra elemental muestra que, en el caso sencillo que estamos refiriendo (un producto y un insumo), las cuatro formulaciones resultan idénticas, es decir:

$$
\pi(1)=\pi(2)=\pi(3)=\pi(4)
$$

Las cuatro formulaciones anteriores de la productividad, y su equivalencia, sólo son válidas por definición en el caso sencillo señalado. En la práctica, sin embargo, las empresas generan más de un producto y utilizan más de un insumo. Y lo mismo sucede, en términos generales, cuando se trasciende la unidad de producción y se intenta cuantificar la productividad para un conjunto de empresas dentro de una industria, o entre industrias a escala de la economía en su conjunto. Para esos casos (múltiples productos o múltiples insumos) estas formulaciones para la medición de la productividad no siempre resultan equivalentes, porque va de por medio el problema de su agregación, y esa agregación debe llevarse a cabo de manera sistemática, lo que necesita de la aplicación de números índices, como veremos a continuación.

\section{UTILIZACIÓN DE NÚMEROS ÍNDICES}

En su argumentación original, Solow (1957) sugirió implícitamente la necesidad de utilizar números índices para la medición del "cambio tecnológico", es decir, la versión de la productividad multifactorial que dio lugar a la contabilidad del crecimiento. Para ello utilizó un índice de tipo Divisia, cuya particularidad reside en considerar el tiempo en forma continua, esto es, como movimientos infinitesi- 
males entre cualesquiera dos momentos dados. ${ }^{11}$ Esto es así porque la medición se plantea en términos de tasas de crecimiento calculadas como resultado de diferenciar las variables respecto del tiempo en la medición de la productividad multifactorial. ${ }^{12}$ Ello no pasó desapercibido para los especialistas, quienes han argumentado que, en términos estadísticos, la información existente considera el tiempo en forma "discreta", es decir, no se dispone de toda la información que cabria esperar entre dos momentos determinados, lo que da lugar a la necesidad (rigurosa) de plantear el problema en términos de números índices ordinarios, es decir, los basados en una consideración discreta del tiempo. ${ }^{13}$

Los números índices más comúnmente empleados en la agregación de cantidades heterogéneas de insumos y de productos, son los denominados de Laspeyres, Paasche y Fisher. Como se sabe, el primero utiliza ponderaciones fijas del año base y el segundo ponderaciones del periodo corriente, en tanto que el índice (Ideal) de Fisher constituye un promedio geométrico de los dos anteriores.

En principio, todo lo que se necesita para la medición de la productividad, en el caso más general de varios productos y varios insumos $(n>1 ; m>1)$ de acuerdo con la segunda formulación $[\pi(2)]$ planteada en el inciso anterior, es contar con índices de cantidad de productos y de insumos, ya que el cociente del primero entre el segundo da una medición de lo que se denomina productividad multifactorial. ${ }^{14}$ Sin embargo, para calcular la productividad mediante las demás

\footnotetext{
${ }^{11}$ En efecto, su formulación se plantea como la derivada de las variables con respecto del tiempo, lo que implica una consideración continua del tiempo.

${ }^{12}$ Siguiendo a Solow, numerosos investigadores han utilizado extensamente el índice Divisia para propósitos de medición de la productividad multifactorial, entre otros Denison (1967), Kendrik (1961), Jorgenson y Griliches (1967), y Christensen y Jorgenson (1967).

${ }^{13} \mathrm{El}$ problema de fondo, se supone, es que bajo el planteamiento de tiempo continuo implicado por el índice Divisia, la separación discreta de la información implica el llamado problema del sendero de dependencia (Hulten, 1973). Ello quiere decir que entre dos momentos dados las mediciones de insumos, productos y productividad pueden adoptar una infinidad de trayectorias, por lo cual se requeriría determinar con exactitud la secuencia especifica que sigue cada una de las variables, esto es, las mediciones de hoy dependen de las mediciones de ayer, si lo que se busca es llegar a cubrir la distancia que media entre el tiempo " $t$ " y el " $t+1$ " por ejemplo. En términos empíricos significa que el índice de Divisia no satisface la prueba de "circularidad", es decir, que si se calcula el crecimiento exponencial de la productividad entre el primero y el último año del periodo analizado, el resultado no es necesariamente igual al promedio de las tasas anuales de crecimiento entre los años extremos, esto es, lo que sucede en los años intermedios no se captura mediante la aplicación del índice Divisia. Fisher (1927), por su parte, argumenta convincentemente que la prueba de circularidad no es esencial para que se tenga un número índice satisfactorio, y Färe et al. (1994b) indican que ello sólo se cumple (en las mediciones de productividad) si el cambio tecnológico es Hicks-neutral.

${ }^{14}$ Suele llamarse también Productividad Total de los Factores, lo que en rigor resulta muy pretencioso porque casi nunca es posible considerar la totalidad de los insumos en las mediciones y, lo que es más, los insumos suelen referirse la mayoría de las veces sólo al capital y a la mano de obra y, en algunas circunstancias, se incluyen los insumos Intermedios.
} 
formulaciones se hace necesario tomar en consideración, -además de índices de cantidades- índices de precios, tanto de insumos como de productos. De hecho, los índices de precios de Laspeyres, Paasche y Fisher son muy similares a los utilizados para agregar cantidades, pero revirtiendo el papel de las cantidades y los precios en cada una de las formulaciones.

Un índice de precios constituye la contraparte de un índice de cantidades, siempre y cuando se satisfaga la llamada regla del producto, esto es, que la multiplicación del índice de cantidad por el de precios, en el caso de los insumos, resulte igual al índice de crecimiento de los costos totales y, en el caso de los productos, resulte igual al índice de crecimiento del ingreso total percibido por la empresa. ${ }^{15}$ Estas referencias elementales nos permiten plantear los casos en los cuales la utilización de números índices satisface las diferentes formulaciones para la medición de la productividad enumeradas en el inciso anterior para el caso más sencillo de un producto y un insumo.

En el caso más general de la empresa multiproductora que utiliza diversos insumos, es posible calcular el crecimiento de la productividad multifactorial si se emplean números índices de acuerdo con las diversas formulaciones expresadas anteriormente, sólo bajo algunas condiciones restrictivas. Diewert y Nakamura (2003) demuestran que, dada una selección del tipo de índice de cantidades (Laspeyres, Paasche o Fisher) tanto para productos como para insumos, las formulaciones primera, segunda y tercera para la medición de la productividad son equivalentes, es decir $\pi(1)=\pi(2)=\pi(3)$, y generan índices válidos y equivalentes del crecimiento de la productividad. Sin embargo, la cuarta formulación $[\pi$ (4)] necesita de algunas restricciones, y éstas están dadas por el tipo de índice de precios que es funcional con los diversos índices de cantidades (de insumos y productos) utilizadas en el cálculo, según los requerimientos para cumplir con la regla del producto ya mencionada.

Así, si $\pi(4)$ aplica índices Paasche de cantidades, la equivalencia con las demás formulaciones solo será válida en caso de que los respectivos índices de precios adopten la forma Laspeyres; por el contrario, si los de cantidades son del tipo Laspeyres, los de precios deben ser del tipo Paasche, y si los de cantidades son del tipo Fisher, los índices de precios deben ser también de tipo Fisher. Sólo bajo

\footnotetext{
${ }^{15}$ Por lo general la contraparte de los índices de cantidad y de precio, para cumplir con la regla del producto, no pueden ser del mismo tipo. Por ejemplo, si el índice de producto es del tipo Paasche, el correspondiente de precios que cumple con la regla debe ser del tipo Laspeyres. A la inversa, los índices de cantidades de tipo Laspeyres deben tener como contraparte índices de precios de tipo Paasche para cumplir con la regla del producto. La excepción es el índice de Fisher, que cumple con tal regla si ambos índices (cantidades y precios) son del tipo (Ideal) de Fisher.
} 
esas condiciones se garantiza la equivalencia de todas las formulaciones descritas para la medición de la productividad, esto es: $\pi(1)=\pi(2)=\pi(3)=\pi$ (4). Esta ultima formulación $[\pi(4)]$ evita las restricciones señaladas sólo en el caso en que el margen de ganancia sea nulo, es decir, cuando $\mathrm{m}_{\mathrm{t}}=0$, en cuyo caso la productividad multifactorial puede medirse como el cociente del índice de precios de los insumos dividido por el índice de precios de los productos, lo que constituye la esencia del planteamiento "dual" de medición de la productividad, que es una variante de la aplicación de los números índices. ${ }^{16}$

Vale apuntar, por último, que otro tipo de índice ha sido también muy utilizado para la medición del crecimiento de la productividad. Éste se conoce como el índice de cantidades de Törnqvist o índice translogarítmico, que se expresa en términos logarítmicos, y cuyas ponderaciones constituyen el promedio de las ponderaciones de dos años consecutivos, suele considerarse como la versión del índice Divisia que se aproxima a tiempo discreto (Jorgenson y Griliches, 1967). ${ }^{17}$ La experimentación numérica detallada ha dejado en claro que, en términos empíricos y estadísticos, la aplicación de este índice proporciona resultados muy similares a los que se obtienen aplicando el índice de Fisher, por lo que, dada la facilidad con que se instrumenta, estadísticamente se recomienda la aplicación del primero para aproximar el resultado del segundo (Diewert, 1978; Hill, 2000). ${ }^{18}$

Tanto en términos teóricos como empíricos tenemos, entonces, diversas alternativas de números índices para seleccionar los más adecuados, con el objeto de cuantificar la productividad multifactorial en el caso de empresas (industrias) multiproductos y utilizadoras de varios insumos. La pregunta es: ¿cuál seleccionar? Varios especialistas se han hecho esta pregunta y, en términos generales, han adoptado dos enfoques diferentes para responderla: a) el enfoque axiomático y b) el enfoque económico.

16 El margen de ganancia puede ser nulo por numerosas razones, pero la más comúnmente invocada es que tal margen es igual a cero por necesidad cuando se supone la existencia de rendimientos constantes a escala y existencia de mercados perfectos, tanto de productos como de factores. Es por ello que ambos supuestos resultan fundamentales para la aplicación del enfoque dual para la medición de la productividad multifactorial, el cual en efecto constituye un caso particular de la formulación $\pi$ (4).

17 En términos sencillos, el índice Törnqvist expresa el promedio geométrico ponderado de las tasas de crecimiento de datos micro-económicos de cantidades. Como las tasas de crecimiento se calculan por diferencia del logaritmo de las cantidades originales, de ahí su nombre de translogarítmico. Los índices de precios translogarítmicos que cumplen con la regla del producto tienen que calcularse de manera implícita, esto es, como el cociente del índice de ingresos totales (costos totales) divididos por sus correspondientes índices translogarítmicos de cantidades (productos o insumos) (Diewert, 1992).

18 La necesidad de aproximar el resultado de aplicar índices de Fisher por medio de índices de Törnqvist es de carácter empírico: la disponibilidad de información para el cálculo del segundo es más accesible que la requerida para calcular el primero. 
En el primer enfoque, los teóricos de los números índices han sugerido diversas propiedades matemáticas mutuamente consistentes que, sobre bases apriorísticas los índices de precios deben reunir propiedades cuyo cumplimiento permite seleccionar de manera univoca la formulación más apropiada. Hecha la selección, se aplica la regla del producto para seleccionar el índice de cantidades correspondiente. En nuestro caso esa selección permite, además, elegir la forma funcional más adecuada del índice de crecimiento de la productividad multifactorial. No nos detendremos aquí en las discusiones técnicas implicadas por este enfoque, y sólo nos referiremos a los resultados que han sido reiteradamente obtenidos por los especialistas. ${ }^{19}$ En síntesis, se encuentra que sólo el índice de Fisher cumple con los requerimientos axiomáticos planteados, en tanto que las demás formulaciones (Laspeyres, Paasche y Törnqvist) solo cumplen algunas de tales exigencias, aunque por lo general no implican tampoco formulaciones del todo inadecuadas desde el punto de vista estadístico (Diewert, 1976; Funker y Voeller, 1978). ${ }^{20}$

Así, los índices de cantidades y de precios de Fisher constituyen las formulaciones más recomendables desde el punto de vista axiomático, y lo mismo puede decirse del índice implícito para la medición de la productividad, en cualquiera de las formulaciones planteadas en su conceptualización en el apartado previo. Debe recordarse, sin embargo, que el índice de cantidades de Fisher, ante la ausencia de la información requerida, puede ser reemplazado en términos empíricos -y sin incurrir en grandes sesgos- con un índice de cantidades de Törnqvist, ya que ambos generan resultados numéricos muy semejantes (Diewert y Lawrence, 1999: 187).

Debe hacerse notar que, en las discusiones anteriores, no se involucra ningún supuesto de orden económico como base para la selección del índice más apropiado desde el punto de vista empírico. El segundo enfoque, por el contrario, invoca criterios económicos explícitos para la selección de los números índices más pertinentes. Más explícitamente, la selección considera diversos supuestos de comportamiento por parte de los productores, así como criterios sobre la forma que supuestamente asume la tecnología. Diewert (1976) ha demostrado, por ejemplo, que si se supone un comportamiento competitivo (maximizador de ganancias y minimizador de costos) por parte de empresarios tomadores de precios, y si la función de producción adopta la forma translogarítmica, entonces la medición de la productividad puede hacerse bajo la formulación $\pi(2)[n>1 ; m>1]$ si se mide

\footnotetext{
19 Para una discusión más detallada de la aplicación de este tipo de enfoque véase Diewert (1992), y la bibliografía especializada citada por Diewert y Nakamura (2003).

20 Una explicación más terrenal se encuentra en Diewert y Lawrence (1999: 180-187.)
} 
como el cociente de un índice Törnqvist de cantidades de producto dividido por un índice Törnqvist de cantidades de insumos, y ello sin necesidad de realizar la estimación (econométrica) de la función de producción correspondiente. A este tipo de índices los considera Diewert (1976) como números índices exactos.

En una contribución posterior, Diewert (1992) alcanza una conclusión similar para la medición de la productividad multifactorial como el cociente de índices de Fisher de cantidades de productos y de insumos, siempre y cuando se mantengan los supuestos optimizadores en el comportamiento de los empresarios y, además, la tecnología pueda ser representada por una función de producción cuadrática. En términos más generales, la evaluación económica de los números índices apunta la conveniencia de adoptar "agregadores flexibles", es decir, números índices que permitan acceder a funciones de producción doblemente diferenciales, de carácter homogéneo y lineal, que se consideran como números indices superlativos (Caves, Christensen y Diewert, 1982b; Balk, 1998). ${ }^{21}$

En resumen, al examinarse los méritos relativos de los índices de Törnqvist y de Fisher en la medición de insumos, productos y productividad, se concluye que ambos índices pueden justificarse bajo la existencia de supuestos económicos, pero que el de Törnqvist no cumple con todas las pruebas axiomáticas que sí cumple el de Fisher, que además supera las limitaciones propias de los índices Laspeyres y Paasche considerados de manera individual. ${ }^{22}$ Con todo, en términos empíricos no existen razones suficientes para seleccionar uno u otro índice (Törnqvist y Fisher), toda vez que cambian las ponderaciones en los diferentes períodos de estimación, y en términos empíricos generan resultados muy similares (Diewert y Lawrence, 1999).

\section{OTROS MÉTODOS DE MEDICIÓN DE LA PRODUCTIVIDAD}

Otras aproximaciones teóricas y empíricas para calcular los cambios en la productividad multifactorial guardan relación con enfoques alternativos para lograr

\footnotetext{
21 Diewert y Nakamura (2003) señalan que, aún cuando los supuestos de comportamiento económico no se den en la realidad, estas formas de medición de la productividad pueden aún ser seleccionadas, pero sobre la base del criterio axiomático discutido con anterioridad. En ese caso, es sólo la vinculación con la función de producción la que se pierde, no así la interpretación intuitiva de la medición de la productividad por medio de los números índice.

22 Los índices de Laspeyres, al utilizar ponderaciones fijas del año base, incurren en el llamado "sesgo de sustitución", es decir, ponderan con mayor importancia de lo debido los productos (insumos) cuyo crecimiento es menor que el promedio, y con menor importancia relativa los que se acrecientan a tasas mayores que el promedio. Ese sesgo se aminora, sin embargo, si se utilizan índices "encadenados", es decir, si se modifica anualmente la base de ponderación (OCDE, 2001: 82).
} 
su medición. Además de la utilización de los números índices discutida en la sección anterior, suelen privilegiarse tres enfoques adicionales: la utilización de funciones de producción (o de costos) estimadas explícitamente con métodos econométricos, la utilización de funciones de distancia que sustituyen a las funciones de producción, y un cálculo de la productividad que evita los problemas de agregación porque aplica paneles de micro datos a escala de establecimientos y de empresas.

En la base del primer planteamiento, destaca el llamado Teorema de la Función Potencial de Ritcher (1966). Mediante este teorema, se establece que la existencia de mediciones agregadas de productividad necesita de la existencia de una función de producción agregada, si es necesario, a escala de la economía en su conjunto. El problema es que esa función debe resultar de la agregación de las micro funciones de producción lo que exige, a su vez, de supuestos muy restrictivos, ya que requiere que todas las micro unidades de producción de la economía (plantas, empresas, industrias) tengan funciones de producción idénticas, con excepción de un multiplicador constante (Fisher, 1965). ${ }^{23}$ Pese a tales limitaciones conceptuales, es usual que algunos economistas planteen, como método para la medición de la productividad multifactorial, la estimación de funciones agregadas de producción (o de costos) por medio de técnicas econométricas, de manera tal que sea posible identificar los desplazamientos de la función de producción (o de costos) a través del tiempo. ${ }^{24}$

De acuerdo con este enfoque, de carácter paramétrico, la aplicación de métodos econométricos evita la imposición de algunos de los supuestos económicos más restrictivos que suelen hacerse -como por ejemplo el referido a las condiciones de igualación de la productividad marginal con las remuneraciones de los factores-y permite suponer condiciones más generales en otros aspectos, como son los de la existencia de mercados no competitivos, de rendimientos no constantes a escala y de cambio tecnológico no neutral, a la vez que facilita introducir otros refinamientos tales como la inclusión de parámetros de ajuste de costos. Se argumenta a que todo ello permite "explicar" los movimientos del "residuo de Solow".

23 Ello sólo es evitable si las micro funciones de producción son aditivas-separables, como demostró hace mucho tiempo Nataf (1948). En ese caso, las micro funciones de producción son agregables y pueden diferir en más de un argumento entre las empresas.

24 Para una revisión de métodos econométricos para estimar estas funciones de producción véase: Berndt (1991). 
Las "ventajas" que derivan de este enfoque, empero, no parecen anular sus desventajas, tanto en términos teóricos como empíricos. La inviabilidad teórica de la existencia de una función de producción agregada a escala de la economía en su conjunto hace inevitable desechar esta perspectiva, sobre bases puramente teóricas. Además, como suele señalarse (Hulten, 2001; Diewert y Lawrence, 1999), son muy conocidas las fallas que resultan de aplicar el método econométrico en estos y otros casos. Entre otras, destacan las siguientes limitaciones: la obtención de estimaciones extrañas de las isocuantas que de la imposición a priori de restricciones al valor de los parámetros, la abundancia de parámetros a estimar que requiere de muestras muy numerosas de datos, las estructuras altamente flexibles de algunos de los modelos propuestos que reclaman -en ocasiones- de estimaciones no lineales que sólo son válidas bajo condiciones muy especiales y, en general, el siempre presente problema de la simultaneidad de la causalidad entre insumos y productos, que invariablemente induce sesgos de ecuaciones simultáneas. ${ }^{25}$ Berndt (1991), Diewert (1982; 1992), y Fuss y McFaden (1978) señalan, además, los formidables problemas estadísticos que surgen en la selección de una forma funcional viable, tanto para las funciones de producción como para las de costos. En suma, las "ventajas" de los enfoques paramétricos tienen implícito un costo que, cuando mucho, sería aceptable si en la práctica estas estimaciones se acompañasen de cálculos no paramétricos que establezcan los límites de validez de las estimaciones de ese tipo. ${ }^{26}$

Invariablemente, los enfoques que intenten equiparar los cambios en la productividad multifactorial con el cambio tecnológico, tienen que suponer que las unidades productivas son técnica y asignativamente eficientes. Si no se tiene tal pretensión, y se sabe que no todas las empresas son técnicamente eficientes, entonces los cambios en la eficiencia tendrían, por necesidad, que reflejarse en las mediciones de productividad. Bajo esas premisas, entonces, otros investigadores han tratado de descomponer los cambios de la productividad multifactorial en dos componentes principales: a) cambio tecnológico, y b) cambios en la eficiencia (técnica y asignativa) de las empresas. Para ello se utiliza el enfoque de la frontera de producción, que constituye la tecnología que representa "las mejores prácticas” (Salter, 1966), es decir, el benchmarking contra el cual se compara la

25 Para una evaluación detallada de los pros y contras de la aplicación del método econométrico en la medición de la productividad multifactorial véase Shreyer y Pilat (2001).

26 Así, por ejemplo, Nadiri y Prucha (2001) combinan los dos enfoques: el paramétrico (econométrico) y el no paramétrico (números índices) para desagregar los resultados de la productividad multifactorial en términos de los efectos de las economías de escala, los costos de ajuste de los insumos factoriales, las innovaciones técnicas, las tendencias no clasificadas y los errores de medición. 
eficiencia de los productores individuales. En este enfoque, los productores que operan en la frontera de producción son técnicamente eficientes, y los que operan por debajo de ella son técnicamente ineficientes.

La bibliografía especializada da cuenta de dos métodos diferentes para lograr la separación de ambos componentes del cambio en la productividad multifactorial. En el primero, la función de producción frontera se estima por métodos econométricos ad hoc, bajo un enfoque de carácter paramétrico; en el segundo, la frontera de producción se estima por métodos no paramétricos, mediante la aplicación de la función distancia.

El enfoque paramétrico, originalmente sugerido por Aigner, Lovell y Schmith (1977), plantea una función de producción (o de costos) en la que el término de error tiene dos componentes: uno estocástico y otro sistemático, y este último captura los efectos de la ineficiencia técnica. ${ }^{27}$ En el enfoque no paramétrico, por el contrario, la frontera de producción que sirve de base para la aplicación de la función distancia, se calcula por medio de algoritmos de programación lineal, lo que permite descomponer el crecimiento de la productividad en los dos componentes mencionados. En este caso, la medición de la productividad multifactorial constituye la media geométrica de dos índices del tipo Malmquist, calculados sobre la base de la función distancia que separa a las observaciones (productores) de la frontera estimada de producción en cada periodo (año) analizado. Este método permite, entonces, envolver los datos para identificar las mejores prácticas de producción y sus desplazamientos en el tiempo (cambio tecnológico), y permite distinguirlos de los cambios en el grado de ineficiencia con que operan las empresas y que pueden derivar de muy diversos factores, como por ejemplo, cambios en la utilización de la capacidad, cambios en la estructura de la economía, cambios en los efectos de regulaciones que inciden en la competitividad, entre otros (Färe, et al. 1985) ${ }^{28}$ Bajo algunos supuestos restrictivos, Balk (1993) demuestra que los resultados de aplicar los índices de Malmquist para la medición de la productividad multifactorial a través de las funciones distancia pueden llegar a ser equivalente (mas no idénticos) de los que resultan de la aplicación de los índices de Törnqvist referidos mas arriba, pero los supuestos en todo caso son bastante restrictivos. ${ }^{29}$

27 Estimaciones equivalentes pueden derivarse de la estimación de funciones frontera de costos y/o de maximización de ganancias, enfoques ambos que además permiten incluir varios productos en las estimaciones (Kumbhakar y Lovell, 2000: Cap. 8).

28 En términos empíricos, este enfoque ha tomado relevancia a través de la aplicación del programa de computo denominado Data Envelopment Analysis (DEA). (Vease Coelli et al., 1998).

29 Esos supuestos son: a) existencia de rendimientos constantes a escala, b) los productores maximizan ganancias, y c) todas las empresas son técnica (y asignativamente) eficientes. 
Aunque el intento por distinguir los efectos del cambio tecnológico (puro) de los derivados del cambio de la eficiencia de los productores en la medición de la productividad multifactorial es, en sí mismo, muy interesante y está sujeto a diversas críticas por parte de los especialistas. En el caso de las fronteras estocásticas, su estimación resiente las ya mencionadas críticas a la aplicación del método econométrico para la estimación de funciones de producción tradicionales. La utilización de técnicas no paramétricas, por otra parte, es muy sensible a la existencia de observaciones atípicas (outlyers) que sesgan con frecuencia la estimación de la envolvente de los datos por medio de algoritmos de programación lineal (oCDE, 2001: 89).

Por último, y dado el creciente acceso que algunos especialistas han logrado a los microdatos de censos y encuestas, en la última década ha tendido a privilegiarse el análisis de los determinantes de la productividad microeconómico, esto es, de plantas o de empresas individuales, mediante la utilización de bancos de datos tipo panel que contienen información de las unidades productivas que operan en diferentes períodos..$^{30}$ Una detallada revisión de los principales estudios realizados con este tipo de información pone de manifiesto que los problemas de medición de la productividad descritos hasta ahora, se multiplican considerablemente en la aplicación de este enfoque. En efecto, Bartelsman y Doms (2000) destacan, por ejemplo, que al aplicarse el concepto de la productividad multifactorial a escala microeconómica, ésta suele medirse como el crecimiento del cociente que resulta de dividir los productos por alguna medida combinada de los insumos utilizados por los establecimientos, lo que implica la necesidad de utilizar números índices a escala desagregada, tanto de productos como de insumos.

Las estimaciones de la productividad multifactorial, tanto en términos individuales como en relación a algún agregado de empresas, enfrenta por lo mismo problemas similares a los ya referidos más arriba. Los mayores problemas, sin embargo, surgen de la medición tanto de productos como de

\footnotetext{
${ }^{30}$ Estos bancos de datos, denominados LMDs por sus siglas en Inglés (Longitudinal Micro-Level Data sets), están constituidos con información de muy grandes números de empresas y/o establecimientos, las cuales son identificables individualmente a lo largo del tiempo. Su utilización para la investigación empírica se ha dirigido a una multiplicidad de tópicos, entre los que destaca el análisis de la productividad. El empleo de esta información masiva, posible sólo en medios electrónicos, deriva de la disponibilidad de los micro datos de censos y encuestas. Se dispone de LMDs tanto en Estados Unidos y Canada, como en otros países europeos. En México se cuenta con una muestra (no aleatoria) contenida en la Encuesta Industrial Anual elaborada por el INEGI, que cubre una decena de años solamente. Hernández Laos (1985) utilizó los micro datos del censo industrial de México en el análisis de productividad, pero esa información no tiene el carácter longitudinal a que se refiere el enfoque aquí comentado.
} 
insumos, especialmente con las de los acervos (o los servicios) de los bienes de capital. En este grado de desagregación cobra especial importancia, por otra parte, el supuesto de la existencia (o no) de mercados competitivos, toda vez que en la deflación para calcular índices de cantidades en el tiempo se aplican índices de precios estimados a escala de industrias enteras, por lo que la no observancia del supuesto refleja las diferencias de precios entre las empresas en las micromedidas de productividad. La bibliografía especializada para este tipo de análisis ha utilizado, indistintamente, alguno de los enfoques ya mencionados de medición: aplicación de índices Divisia aproximados con índices Törnqvist; estimación econométrica de funciones de producción (o de costos), por citar algunos. Se reconoce, empero, que los problemas de la simultaneidad de la causalidad entre insumos y productos se acentúa considerablemente al aplicarse los enfoques econométricos a los micro datos.

En general, los especialistas que han aplicado con mayor interés este enfoque se concentran más en la discusión de los resultados a los que llegan en sus análisis, que en los problemas implícitos en la medición de la productividad, a pesar de que tales problemas suelen ser considerables en este nivel micro económico, como ya se ha mencionado. Habría que añadir, además, la muy escasa disponibilidad de este tipo de microinformación para largos periodos, lo que inhabilita a este enfoque para hacer análisis de largo plazo.

\section{MÉTODO DE MEDICIÓN RECOMENDADO}

El planteamiento original del residual de Solow se convirtió, con los años, en una herramienta de análisis del crecimiento económico que se utiliza indiscriminadamente en trabajos empíricos. El problema es que tienden a pasarse por alto los muy restrictivos supuestos que implican la validez de la interpretación originalmente dada por su autor, en el sentido de suponer que tal residual mide los desplazamientos de la función de producción y que, mediante estos, se busca una aproximación para la medición del cambio tecnológico. Sin embargo, en la base misma de tal concepción radica un problema conceptual de carácter muy complejo. En efecto, desde hace ya varias décadas, en un muy poco citado trabajo de Atkinson y Stiglitz (1969) se explica por qué en rigor, no tiene sentido identificar el avance tecnológico con los desplazamientos de la función de producción. En efecto, argumentan que las mejoras tecnológicas afectan procesos específicos y no tendrían -en principio- por qué desplazar toda la función de producción como supone el 
argumento de Solow, sino sólo en el punto en que la empresa (o la economía) está operando. ${ }^{31}$

Pero no solamente en términos conceptuales resulta poco satisfactorio identificar el cambio tecnológico con los desplazamientos de la función de producción, sino que en términos empíricos resulta muy restrictivo tratar de estimarlo a partir del acceso a información sólo de precios y cantidades de insumos y productos. El costo, como hemos visto, es el de suponer condiciones muy improbables, tanto sobre el comportamiento optimizador de los productores, como en relación con la existencia de rendimientos constantes a escala y, en general, en relación a condiciones optimas desde el punto de vista del sistema económico en su conjunto. Supuestos que si bien pudieran darse -y sólo en alguna medida- en algunas economías industrializadas, resultan muy difíciles de observar en los países en desarrollo donde las fallas de los mercados son de carácter generalizado y muy difícilmente se alcanzan condiciones optimas de Pareto (Hoff y Stiglitz, 2001). ${ }^{32}$

Ante ello, cabe preguntarse: ¿resulta razonable hacer esos supuestos a sabiendas de que difícilmente se observan en la realidad? Desde luego que no, y es por ello que el enfoque económico de la tradición neoclásica -basado en criterios optimizadores para medir la productividad multifactorial- debería abandonarse y adoptarse otro que no resulte tan oneroso en términos de su-

\footnotetext{
${ }^{31}$ En realidad -argumentan Atkinson y Stiglitz- aunque podría esperarse que un avance tecnológico determinado genere algunos desbordamientos (spillovers) que afecten a otras técnicas de producción, es improbable que incidan en todas y cada una de las técnicas especificadas por la función de producción. Es, para poner un ejemplo, como si de una mejora específica en algún tipo de huso textil se viesen afectados indiscriminadamente todos los tipos de husos textiles, desde los de carácter manual hasta los más automatizados. En todo caso, las mejoras tecnológicas -más allá de los desbordamientos posibles- tenderían a modificar la forma de la función de producción, específicamente en la técnica en la cual la mejora tiene lugar, pero es altamente improbable que afecte a todas las técnicas y desplace toda la función de producción como supone el argumento neoclásico que, por cierto, deriva de planteamientos del mismo Hicks.

32 Para la teoría neoclásica las instituciones particulares de una economía no son relevantes, toda vez que suponen que el desempeño económico es resultado de fuerzas fundamentales representadas sólo por los recursos, las preferencias y las tecnologías, cuya interacción conduce a situaciones de óptimas de Pareto en las que las instituciones no logran influenciar la selección del equilibrio. Sin embargo, como lo demuestran Hoff y Stiglitz (2001), la interacción derivada de las instituciones prevalecientes en los países en desarrollo es capaz de generar comportamientos que pueden conducir a equilibrios múltiples y cada uno ser ineficiente. La interacción de tales instituciones con los patrones prevalecientes de distribución de la riqueza, la historia y la ecología, condicionan el alcance de la eficiencia, por la existencia de costos elevados de transacción y problemas de información asimétrica que pueden conducir a equilibrios múltiples, en los cuales tanto las preferencias como las tecnologías y las instituciones son todas endógenas y, por lo tanto, eventos transitorios pueden tener efectos duraderos en ellas y en los equilibrios resultantes. Alcanzar un único equilibrio, de carácter óptimo en el sentido paretiano, es muy improbable en los países subdesarrollados, dadas las fallas generalizadas en la operación de los mercados, producto, entre otros factores, de instituciones ineficientes.
} 
puestos poco realistas. Hemos visto cómo algunos especialistas afirman que el enfoque econométrico de las funciones de producción no implica la existencia de algunos de esos supuestos, aunque las limitaciones conceptuales y las dificultades del método estocástico desaconsejan -como ya vimos- su aplicación, especialmente por las complejidades que derivan de la simultaneidad de la causalidad entre insumos y productos. Queda, por tanto, la utilización de algún método más pragmático que no implique los mencionados supuestos, y ese método parece ser consistente con el enfoque axiomático para la selección de números índices.

Por ello, en investigaciones empíricas recomendamos el uso del enfoque que emplea números índices para la medición de la productividad multifactorial. En efecto, proponemos un índice que parte de la igualdad contable, en un periodo dado, entre los ingresos y los costos de producir cualquier producto "i”, incluyendo las ganancias "anormales" (si las hubiere). Por medio de la valuación de los recursos a utilizar en los siguientes periodos a los precios del periodo previo, y mediante un proceso de agregación de funciones lineales de todos los productos (de la industria o de la economía), es posible llegar a una medición de la productividad multifactorial sin necesidad de incurrir en supuestos optimizadores (maximización de ganancias y/o minimización de costos), medición que es consistente en términos estrictos con la aplicación de la teoría de los números índices. En el anexo, al final de este texto, se detalla el desarrollo matemático para llegar a esta definición de la productividad multifactorial, que se expresa como el cociente de dos números índices de cantidad: uno de productos dividido por otro de insumos factoriales, lo que es consistente con la formulación $\pi$ (2) para la medición de la productividad referida más arriba. ${ }^{33}$

Dada la ausencia de supuestos sobre el comportamiento optimizador de los agentes en nuestra formulación, así como sobre la existencia de rendimientos a escala, las mediciones de la productividad multifactorial deben incorporar, por necesidad, no sólo los efectos de las innovaciones tecnológicas,

\footnotetext{
33 Aunque -como se explica en el Anexo- el índice implícito en esta formulación es del tipo Laspeyres, la reformulación del problema cambiando la base del planteamiento conduce a un índice del tipo Paasche. Si el cálculo de ambos se hace mediante encadenamientos anuales, esto es, cambiando anualmente la base, es posible derivar un índice (Ideal) de Fisher mediante el promedio geométrico de los dos índices anteriores, índice el de Fisher que es el más adecuado si nos atenemos a las recomendaciones del enfoque axiomático para la selección de números índices. Ahora bien, dado que en términos empíricos este índice genera resultados muy aproximados a los que produce la aplicación de índices del tipo Törnqvist, y dada la facilidad de instrumentación de este último en términos estadísticos, se recomienda que en las aproximaciones empíricas se aplique esta última formulación, y todo ello por conveniencia estadística y sin necesidad de incurrir en supuestos económicos poco realistas sobre el comportamiento de los mercados y los agentes.
} 
sino también los cambios (no observados) de la eficiencia técnica y asignativa de los productores. Por ello, antes de proceder al trabajo empírico cabe, sin embargo, preguntarse ¿qué interviene en los movimientos de la productividad multifactorial? ¿cuál es el significado de sus movimientos y modificaciones a lo largo del tiempo?

SUMARIO: EL SIGNIFICADO DE LA PRODUCTIVIDAD MULTIFACTORIAL

Para los primeros analistas de la productividad multifactorial, ésta constituía tan sólo una "medida de nuestra ignorancia" (Abramovitz, 1956), dado su carácter empírico y su forma de cálculo como el residuo resultante de la diferencia entre las tasas de crecimiento del producto y de los insumos. Al vincular su formulación meramente empírica con una estructura teórica congruente, Solow (1957) avanzó un paso en la identificación del significado de este residuo, y lo relacionó con los desplazamientos de la función de producción derivados del "avance tecnológico". El costo, sin embargo, como ya hemos visto, resultó muy oneroso en términos de supuestos sobre el comportamiento optimizador de los productores, la existencia de rendimientos constantes a escala y la presencia de mercados competitivos tanto de productos como de factores. Los esfuerzos analíticos de los investigadores se dirigieron así, durante varias décadas, a la discusión de las formas más apropiadas para cuantificar los cambios en la productividad multifactorial, pero sin abandonar de una manera u otra la pretensión de que lo que estaban midiendo era el avance tecnológico.

Discusiones posteriores trataron de precisar qué tipo de cambio tecnológico se estaba midiendo con las cuantificaciones de la productividad multifactorial. Resultó más o menos claro que -aún bajo los supuestos dominantes- ésta no reflejaba todo el cambio tecnológico, sino sólo la parte no "onerosa" de los avances técnicos (Jorgenson y Griliches, 1967), lo que más tarde se conocería como las "tortas gratis" (free lunches), "maná del cielo" y otras denominaciones parecidas, porque en una variedad de situaciones el cambio tecnológico queda incorporado mediante nuevos diseños y calidades, en las nuevas generaciones de los bienes de capital y de los insumos intermedios. En la medida en que los insumos estén bien medidos, ese cambio tecnológico incorporado quedaría expresado en el crecimiento de los insumos de capital y no en el de la productividad multifactorial. Otro tipo de cambio tecnológico, el de carácter desincorporado que resulta de avances en la ciencia en los diseños y las formulaciones, así como de la difusión del conocimiento -incluidos los avances en los procesos admi- 
nistrativos y organizacionales- y cuya transmisión no requiere de transacciones onerosas en el mercado, sí se debería reflejar en los cambios de la productividad

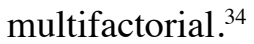

Sin embargo, se concluía, si en la medición de la productividad se utilizan medidas no ajustadas de los insumos de capital y mano de obra, se esperaría que aquella incluyese tanto los efectos del cambio tecnológico incorporado como los del no incorporado, por lo que el significado de la productividad multifactorial depende, en todo caso, de la manera como se mide. Lo correcto -teórica y empíricamente- era medirla e interpretarla -bajo todos los supuestos usuales- como efecto sólo del avance tecnológico no incorporado. ${ }^{35}$

La rigidez de los supuestos -sobre todo el de la existencia de mercados competitivos- llevó a ampliar el significado de la productividad multifactorial. Hall (1998) demostró que, en condiciones de competencia imperfecta, el precio es mayor que el costo marginal, o en presencia de rendimientos crecientes a escala, la productividad multifactorial incorpora algunos cambios en la magnitud de los insumos variables, así como las ganancias anormales contenidas en el sobreprecio (mark-up) que fijan las empresas oligopólicas. Es decir, el residuo de Solow deja de convertirse sólo en un desplazamiento neutral de la función de producción, para convertirse en una expresión combinada de éste y de los rendimientos crecientes.

Al seguir esa línea de razonamiento, y considerar el hecho de que toda innovación conlleva un costo de gestación, Carlaw y Lipsey (2003) han afirmado que lo que la productividad multifactorial refleja, en todo caso, son las ganancias anormales derivadas de la innovación tecnológica en condiciones de incertidumbre, esto es, ganancias inesperadas que se reparten entre productor y comprador dependiendo del tipo de mercado. ${ }^{36}$ Pero ni siquiera esas ganancias anormales se capturan completamente en las mediciones de la productividad multifactorial, sobre todo en situaciones en las cuales el reza-

\footnotetext{
34 Otros efectos que tienen el carácter de desbordamientos (spillovers) derivados del capital, la mano de obra y los insumos intermedios, y que constituyen adiciones no onerosas a la productividad en su conjunto generadas por ciertos tipos de trabajo o de capital, deberían reflejarse también en las mediciones de la PTF. 35 Para Jorgenson (1995), por ejemplo, la productividad multifactorial debe reflejar sólo los efectos sobre el crecimiento del producto que no constituyen inversión, es decir, aquellos para los cuales la empresa no compromete recursos para aumentar las expectativas de ingresos futuros (free lunches.)

36 Estas ganancias anormales asociadas con el cambio tecnológico se constituyen por la suma de todos los aumentos de producción y/o de reducción de costos que se dirigen a cualquier agente de la economía, menos el costo respectivo de desarrollar la nueva tecnología. La productividad multifactorial, en todo caso, incluye esa parte del retorno de las innovaciones que es una recompensa por la incertidumbre y, en esa medida, es parte de los free lunches de Jorgenson.
} 
go de los desbordamientos no es considerado en la medición de los productos y los insumos corrientes. ${ }^{37}$

Todas las interpretaciones anteriores, de una $\mathrm{u}$ otra manera, suponen un comportamiento optimizador por parte de los productores y, por lo mismo, se supone que operan en niveles máximos de eficiencia técnica o asignativa. Hemos visto más arriba, sin embargo, que en la realidad este supuesto no se sostiene $\mathrm{y}$, por tanto, los movimientos de la productividad multifactorial deben dejar de ser interpretados sólo como reflejos del cambio tecnológico y de las ganancias anormales asociadas con las innovaciones de la tecnología para incluir, por necesidad, los efectos de cambios en los niveles de eficiencia de los productores. Este reconocimiento llevó a la introducción de los enfoques para la medición de la productividad multifactorial que permiten distinguir, en términos empíricos, entre el cambio tecnológico (desplazamientos de la frontera de producción) y los efectos de modificaciones en las condiciones medias de eficiencia de los productores (movimientos desde o hacia la frontera de producción) (Färe et al., 1988, 1994a).

Sin proponérselo, y en un contexto discursivo diferente, Harberger (1998) allanó el camino para considerar los aumentos de la productividad multifactorial desde este punto de vista más amplio, en el que es posible incorporar tanto los efectos del cambio tecnológico, como en las ganancias anormales asociadas a éste y, paralelamente, considerarlos como efectos de los cambios en las condiciones de eficiencia de los productores. Para Harberger, los cambios en la productividad multifactorial son resultado de numerosos sucesos y factores que ocurren simultáneamente en cada momento. Es decir, los considera como "un paraguas que cubre las reducciones reales de costos de todo tipo (RCR) que adoptan mil y un formas que difícilmente pueden representarse por una, dos o tres variables agregadas" (Harberger, 1998: 20). ${ }^{38}$

\footnotetext{
37 Esos casos son los siguientes: a) cuando los efectos de las reducciones de costos toman tiempo para realizarse y convertirse en ganancias; b) al contabilizarse el gasto en I\&D como insumo intermedio, pero no la ganancia derivada de ese gasto; c) cuando se omiten insumos, como por ejemplo los recursos naturales, o las inversiones informales en I\&D; d) cuando no se cuantifican correctamente los insumos, lo que hace que los cambios (no medidos) en la calidad de los insumos se refleje como cambio en la productividad multifactorial; e) cuando la economía toma tiempo para ajustarse a los cambios en la tecnología, y f) cuando los desbordamientos son de carácter rezagado, como sucede en el caso de las tecnologías de aplicación generalizada (General purpose technologies) (Helpman, 1998), las cuales, al diseminarse, hacen que no correspondan al período corriente de comparación entre productos e insumos, sino que se disperse en toda la economía durante muchos períodos posteriores.

38 Esta forma de considerar los efectos del cambio tecnológico sobre la productividad y su efecto en la reducción de costos reales se remonta al libro seminal de Salter (1966), y tiene su expresión cuantitativa en las mediciones de la productividad multifactorial a través del método "dual," planteadas desde hace varias décadas por Siegel (1961) y Lydall (1969).
} 
Para Harberger, los movimientos de la productividad multifactorial son el resultado de fuerzas que constituyen la dinámica de las economías de mercado, en una visión schumpeteriana de la "destrucción creativa," en cuyo centro se ubican los esfuerzos competitivos de las empresas mediante de la búsqueda incesante de reducción de costos, y cuyos efectos combinados (de disminuciones y aumentos) están implícitos en los procesos de agregación que conocemos en las mediciones de la productividad multifactorial..$^{39}$ Lo fundamental, en todo caso, es reconocer la multiplicidad de fuentes de las que pueden provenir los cambios en la productividad multifactorial considerada como reducciones reales de costos, en un proceso continuo de "perdedores" y "ganadores", en el que los perdedores se caracterizan por disminuciones en el valor agregado real o por tasas de redimiento decrecientes, y los ganadores por mayores niveles de producción -dados unos insumos- y mayores tasas de rendimiento. ${ }^{40}$

La interpretación anterior debe ser tomada en cuenta muy seriamente, si lo que se desea es entender el significado de lo que estamos midiendo bajo el concepto de la productividad multifactorial. Estas mediciones nos permiten cuantificar, de manera consistente y sistemática, una de las fuentes próximas del crecimiento económico que, junto con la acumulación de recursos factoriales, complementa nuestra comprensión del proceso de desenvolvimiento cuantitativo de la economía. En su medición se incorporan, así, la multiplicidad de elementos señalados por Harberger que inciden en la reducción (aumento) de los costos reales de las empresas, y que van desde de los mejoramientos de algunas de las

\footnotetext{
39 Las mediciones tradicionales de la productividad multifactorial se basan en el concepto de equilibrio, que ciertamente no es el más adecuado para examinar los procesos de innovación y crecimiento tecnológico. La tradición evolucionista, seguidora de Schumpeter, sostiene que la innovación y el cambio tecnológico ocurren a consecuencia de asimetrías en la información y, especialmente, en condiciones de imperfecciones de mercado (Foray, 2004.) Según el enfoque evolucionista, si hubiese un equilibrio no surgirían los incentivos para la búsqueda y la innovación y, por lo tanto, no habría cambios en la productividad multifactorial. Es por ello que debe abandonarse el supuesto de la existencia de equilibrio, especialmente del tipo de Pareto óptimo, en las mediciones de la productividad.

40 Los análisis basados en información micro-económica a escala de empresas y/o establecimientos (Foster, Haltinwagner y Krizan, 2001) muestran, en efecto, que hay considerables diferencias en el desempeño de la productividad de las unidades económicas individuales. Además, se muestra que existe una reasignación continua y de grandes proporciones en la producción y los insumos entre empresas, esto es, que el crecimiento de la productividad ocurre a consecuencia de la entrada y salida de empresas, por lo que tiende a estar asociado con la difusión de nuevas tecnologías entre las firmas, más que a consecuencia de desplazamientos simultáneos de producción del conjunto existente de empresas. Debe hacerse notar que ello es consistente con el punto de vista expresado por Atkinson y Stiglitz (1969) citado más arriba, del desplazamiento no de la función de producción completa, sino del movimiento de la frontera de producción a consecuencia de la entrada de tecnologías específicas más productivas y la salida de las menos productivas.
} 
tecnologías disponibles y sus desbordamientos (spillovers) sobre otros procesos productivos, así como sus efectos sobre las ganancias anormales de las empresas. Las mediciones agregadas incorporan, también, los efectos de su diseminación por el sistema económico en su conjunto, las economías de escala, los cambios en la utilización de la capacidad instalada, los cambios en los niveles de eficiencia de las empresas y, por supuesto, los errores de medición de las variables. Destacan, por su importancia, los cambios (puros) de eficiencia (para distinguirlos de los desplazamientos de la frontera de producción) que constituyen un fenómeno empírico por demás común. Todo ello afecta los niveles reales de costo de las empresas, y sus cambios estarían reflejados por los movimientos de la productividad multifactorial.

En la medida en que los procesos condicionantes de la acumulación factorial derivan de un conjunto de causas más o menos identificadas por la teoría del crecimiento, y en tanto que los determinantes de la evolución de la productividad multifactorial así definida derivan de otros factores causales, en ese contexto se justifica explorar la recomendación de los especialistas, cuando afirman que "la contabilidad del crecimiento debe complementarse con estudios históricos, institucionales y de casos, si lo que se busca es explorar algunas de las causas del crecimiento, la innovación y los cambios de la productividad.” (OCDE, 2001: 117). Ese es, precisamente, el enfoque que recomendamos para su adopción. 


\section{ANEXo}

PROPOSICIÓN DE UN ÍNDICE DE PRODUCTIVIDAD MULTIFACTORIAL

Partimos del hecho de que en cualquier periodo " $O$ " el valor de los ingresos totales generados por la producción del producto "i" $\left(Y_{i}\right)$ deben ser iguales al valor total de costos incurridos $\left(\sum_{\mathrm{j}=1 \ldots \mathrm{m}} \mathrm{X}_{\mathrm{ij}}^{0}\right)$, esto es, el valor de la compensación a los " $m$ " factores de producción, incluyendo las ganancias extraordinarias de las empresas (si las hay):

$$
Y_{i}^{0}=\sum_{j=1 \ldots m} X_{i j}^{0}
$$

$\mathrm{Al}$ descomponer tanto el producto como los insumos en su componente de precio y cantidad se tiene:

$$
\mathrm{p}_{\mathrm{i}}^{0} \mathrm{y}_{\mathrm{i}}^{0}=\sum_{\mathrm{j}=1 \ldots \mathrm{m}} \mathrm{w}_{\mathrm{ij}}^{0} \mathrm{x}_{\mathrm{ij}}^{0}
$$

Definimos índices parciales de requerimientos de insumos por unidad de producción, es decir, índices inversos de la productividad parcial de cada uno de los insumos:

$$
\mathrm{H}_{\mathrm{ij}}^{0}=\mathrm{x}_{\mathrm{ij}}^{0} / \mathrm{y}_{\mathrm{i}}^{0}
$$

Y los introducimos en la igualdad anterior, con lo que se tiene una especificación del precio del producto " $i$ ” en el año " 0 ":

$$
\mathrm{p}_{\mathrm{i}}^{0}=\sum_{\mathrm{j}=1 \ldots \mathrm{m}} \mathrm{w}_{\mathrm{ij}}{ }^{0} \mathrm{H}_{\mathrm{ij}}{ }^{0}
$$

Valuamos la producción de un periodo subsiguiente, digamos " 1 ” a los precios del año " $O$ ” y se obtiene:

$$
\mathrm{y}_{\mathrm{i}}^{1} \mathrm{p}_{\mathrm{i}}^{0}=\mathrm{y}_{\mathrm{i}}{ }^{1}\left[\sum_{\mathrm{j}=1 \ldots \mathrm{m}} \mathrm{w}_{\mathrm{ij}}{ }^{0} \mathrm{H}_{\mathrm{ij}}{ }^{0}\right]
$$

Ahora dividimos por el valor del producto " $i$ " en el periodo " 0 ” en ambos lados de la igualdad anterior y se tiene:

$$
\left[\left(\mathrm{y}_{\mathrm{i}}^{1} \mathrm{p}_{\mathrm{i}}^{0}\right) /\left(\mathrm{y}_{\mathrm{i}}^{0} \mathrm{p}_{\mathrm{i}}^{0}\right)\right]=\sum_{\mathrm{j}=1 \ldots \mathrm{m}}\left[\left(\mathrm{y}_{\mathrm{i}}{ }^{1} \mathrm{w}_{\mathrm{ij}}{ }^{0} \mathrm{H}_{\mathrm{ij}}{ }^{0}\right) /\left(\mathrm{y}_{\mathrm{i}}^{0} \mathrm{p}_{\mathrm{i}}^{0}\right)\right]
$$

Ahora bien, dado que: $\mathrm{y}_{\mathrm{i}}{ }^{1}=\mathrm{x}_{\mathrm{ij}}{ }^{1} / \mathrm{H}_{\mathrm{ij}}{ }^{1}$ se sigue que: 


$$
\left[\left(\mathrm{y}_{\mathrm{i}}{ }^{1} \mathrm{p}_{\mathrm{i}}^{0}\right) /\left(\mathrm{y}_{\mathrm{i}}^{0} \mathrm{p}_{\mathrm{i}}^{0}\right)\right]=\sum_{\mathrm{j}=1 \ldots \mathrm{m}}\left[\left(\mathrm{x}_{\mathrm{ij}}{ }^{1} \mathrm{w}_{\mathrm{ij}}{ }^{0}\right)\left(\mathrm{H}_{\mathrm{ij}}{ }^{0} / \mathrm{H}_{\mathrm{ij}}{ }^{1}\right) /\left(\mathrm{y}_{\mathrm{i}}^{0} \mathrm{p}_{\mathrm{i}}^{0}\right)\right]
$$

Al multiplicar y dividir por $\mathrm{x}_{\mathrm{ij}}{ }^{0}$ se tiene:

$$
\left[\left(\mathrm{y}_{\mathrm{i}}^{1} \mathrm{p}_{\mathrm{i}}^{0}\right) /\left(\mathrm{y}_{\mathrm{i}}^{0} \mathrm{p}_{\mathrm{i}}^{0}\right)\right]=\sum_{\mathrm{j}=1 \ldots \mathrm{m}}\left\{\left[\left(\mathrm{w}_{\mathrm{ij}}{ }^{0} \mathrm{x}_{\mathrm{ij}}{ }^{0}\right) /\left(\mathrm{y}_{\mathrm{i}}^{0} \mathrm{p}_{\mathrm{i}}{ }^{0}\right)\right]\left[\left(\mathrm{x}_{\mathrm{ij}}{ }^{1} / \mathrm{x}_{\mathrm{ij}}{ }^{0}\right)\left(\mathrm{H}_{\mathrm{ij}}{ }^{0} / \mathrm{H}_{\mathrm{ij}}{ }^{1}\right)\right]\right\}
$$

Para simplificar la notacion definimos: $\alpha_{\mathrm{ij}}{ }^{0}=\left(\mathrm{w}_{\mathrm{ij}}{ }^{0} \mathrm{x}_{\mathrm{ij}}{ }^{0}\right) /\left(\mathrm{yi}^{0} \mathrm{p}_{\mathrm{i}}^{0}\right)$, en donde $\alpha_{\mathrm{ij}}{ }^{0}$ mide la participación del valor del insumo " $j$ " en el valor del producto “ $i$ ” en el año "O". De manera paralela definimos: $\pi_{\mathrm{ij}}=\mathrm{H}_{\mathrm{ij}}{ }^{0} / \mathrm{H}_{\mathrm{ij}}{ }^{1}$ que muestra el crecimiento de la productividad parcial del insumo " $j$ " en la producción del producto " $i$ " entre el periodo " 0 " y el periodo " $l$ " respectivamente.$^{41}$ De lo anterior se sigue que la última expresión puede escribirse como:

$$
\left[\left(\mathrm{y}_{\mathrm{i}}^{1} \mathrm{p}_{\mathrm{i}}^{0}\right) /\left(\mathrm{y}_{\mathrm{i}}^{0} \mathrm{p}_{\mathrm{i}}^{0}\right)\right]=\sum_{\mathrm{j}=1 \ldots \mathrm{m}}\left(\alpha_{\mathrm{ij}}{ }^{0} \pi_{\mathrm{ij}}\right)\left(\mathrm{x}_{\mathrm{ij}}{ }^{1} / \mathrm{x}_{\mathrm{ij}}{ }^{0}\right)
$$

Al agregar todos los productos " $i$ " $(i=1 \ldots n)$ de la industria (o de la economía) se tiene:

$$
\sum_{\mathrm{i}=1 \ldots \mathrm{n}}\left[\left(\mathrm{y}_{\mathrm{i}}{ }^{1} \mathrm{p}_{\mathrm{i}}^{0}\right) /\left(\mathrm{y}_{\mathrm{i}}^{0} \mathrm{p}_{\mathrm{i}}^{0}\right)\right]=\sum_{\mathrm{i}=1 \ldots \mathrm{n}} \sum_{\mathrm{j}=1 \ldots \mathrm{m}}\left[\alpha_{\mathrm{ij}}{ }^{0} \pi_{\mathrm{ij}}\left(\mathrm{x}_{\mathrm{ij}}{ }^{1} / \mathrm{x}_{\mathrm{ij}}{ }^{0}\right)\right]
$$

Puede demostrarse que, de manera ex post, es posible estimar un promedio ponderado de los escalares $\pi_{\mathrm{ij}}$ para la totalidad de los productos y los insumos, que denominaremos $\pi^{*}$. En el calculo de $\pi^{*}$ las ponderaciones están dadas por la importancia de cada insumo " $j$ ” $(j=1 \ldots m)$ en la elaboración de cada producto $\mathrm{y}$, a la vez, están ponderados por la importancia de cada producto " $i$ " $(i=1 \ldots n)$ en el valor de la totalidad de productos (Hernández Laos, 1985: 152-154). De esta manera, la última igualdad puede expresarse como:

$$
\sum_{\mathrm{i}=1 \ldots \mathrm{n}}\left[\left(\mathrm{y}_{\mathrm{i}}{ }^{1} \mathrm{p}_{\mathrm{i}}^{0}\right) /\left(\mathrm{y}_{\mathrm{i}}^{0} \mathrm{p}_{\mathrm{i}}^{0}\right)\right]=\pi^{*}\left[\sum_{\mathrm{i}=1 \ldots \mathrm{n}} \sum_{\mathrm{j}=1 \ldots \mathrm{m}}\left\{\alpha_{\mathrm{ij}}{ }^{0}\left(\mathrm{x}_{\mathrm{ij}}{ }^{1} / \mathrm{x}_{\mathrm{ij}}{ }^{0}\right)\right\}\right]
$$

El lado izquierdo de la igualdad anterior representa, en esencia, un índice Laspeyres de cantidad del crecimiento del conjunto de los productos $\left(\mathrm{L}_{\mathrm{y}}\right)$ y la ultima parte del lado derecho expresa un índice de cantidad del crecimiento del conjunto de los insumos $\left(\mathrm{L}_{\mathrm{x}}\right)$ en donde cada uno de los insumos esta ponderado por su respectiva participación en el valor de cada producto en el año base. Por lo tanto,

${ }^{41}$ Ello equivale a la formulación $\pi$ (1) de la productividad especificada en el cuerpo del capítulo, correspondiente a cada uno de los insumos utilizados en la producción. 
esa igualdad puede expresarse como:

$$
\mathrm{L}_{\mathrm{y}}=\pi^{*} \mathrm{~L}_{\mathrm{x}}
$$

Y, por lo mismo, se llega a la siguiente expresión:

$$
\pi^{*}=\mathrm{L}_{\mathrm{y}} / \mathrm{L}_{\mathrm{x}}
$$

En donde $\pi^{*}$ expresa un índice de la productividad conjunta de los " $m$ " factores productivos considerados en el análisis, utilizados en la generación de los " $n$ " productos procesados en la industria (economía), y su cuantificación se define como el cociente de dos índices de cantidades de tipo Laspeyres: uno de productos dividido por otro de insumos múltiples. En las agregaciones se utilizan las "participaciones factoriales" $\left(\alpha_{\mathrm{ij}}{ }^{0}\right)$, las cuales no están sujetas a los supuestos económicos tradicionales de comportamiento optimizador por parte de los productores, y tampoco es necesario que en la formulación tenga que suponerse la existencia de rendimientos constantes a escala.

Sabemos las limitaciones a que están sujetos los índices de Laspeyres de cantidades, en el sentido en que, al mantener constante el año base de comparación, se incurre en un sesgo de sustitución conforme avanza el tiempo, considerando con mayor importancia los productos y los insumos que crecen relativamente menos rápido, y restando importancia relativa a los que crecen más aceleradamente. Una forma de atenuar ese sesgo es por medio del encadenamiento de los índices, lo que no es más que cambiar cada año la base de estimación, en cuyo caso el período " 0 " se convierte en el " $t-l$ " y el " $l$ " se convierte en el período " $t$ " a lo largo de toda la serie. Pero el sesgo puede eliminarse totalmente si, en lugar de valuar el producto del año " $t$ " a los precios del periodo " $t-l$ " se valúa el producto del año " $t-1$ " a los precios del período " $t$ " en la tercera igualdad desarrollada anteriormente. En ese caso, se llega a una formulación de la productividad multifactorial similar, pero con base en índices de cantidades del tipo Paasche $\left(\mathrm{P}_{\mathrm{y}}\right.$ y $\left.\mathrm{P}_{\mathrm{x}}\right)$. Se tienen, entonces, para cada par de años sucesivos, dos valores del índice de la productividad, el primero basado en índices de cantidades de Laspeyres $\left(\pi^{*}\right)$ y el segundo basado en índices de cantidades de Paasche $\left(\pi^{+}\right)$, es decir:

$$
\begin{aligned}
& \pi^{*}=\mathrm{L}_{\mathrm{y}} / \mathrm{L}_{\mathrm{x}} \\
& \pi^{+}=\mathrm{P}_{\mathrm{y}} / \mathrm{P}_{\mathrm{x}}
\end{aligned}
$$


Puede entonces, sin perder generalidad el argumento, optarse por el promedio geométrico de ambos índices, lo que generará un índice de productividad multifactorial del tipo (Ideal) de Fisher $\left(\pi^{\mathrm{f}}\right)$, como resultado del cociente de dos índices de Fisher de cantidades, uno de productos $\left(\mathrm{F}_{\mathrm{y}}\right)$ y el otro de insumos $\left(\mathrm{F}_{\mathrm{x}}\right)$ :

$$
\pi^{\mathrm{f}}=\mathrm{F}_{\mathrm{y}} / \mathrm{F}_{\mathrm{x}}
$$

En donde:

$$
\begin{aligned}
& F_{y}=\left[L_{y} P_{y}\right]^{1 / 2} \\
& F_{x}=\left[L_{x} P_{x}\right]^{1 / 2}
\end{aligned}
$$

Esta sería una formulación insesgada (estadísticamente) de la productividad multifactorial, toda vez que como se vio más arriba, es el índice (Ideal) de Fisher el que mejor cumple con los requisitos postulados por el método axiomático de selección de números índices.

Aún más, dado que en términos empíricos el índice de Fisher de cantidades puede aproximarse muy cercanamente por medio de un índice de cantidades de Törnqvist (Diewert y Lawrence, 1999; Diewert y Nakamura, 2003), y dada la relativa facilidad de cómputo de este último tipo de índice, podría ser recomendable llevar a cabo tal aproximación, en cuyo caso se tendría:

$$
\pi^{\mathrm{f}} \approx \pi^{\mathrm{t}}=\left[\mathrm{T}_{\mathrm{y}} / \mathrm{T}_{\mathrm{x}}\right]
$$

Es decir, la productividad multifactorial se aproximaría por el cociente de dos índices de Törnqvist: uno de productos $\left(\mathrm{T}_{\mathrm{y}}\right)$ entre otro de insumos $\left(\mathrm{T}_{\mathrm{x}}\right)$.

Por último, vale hacer notar que toda la discusión anterior continúa siendo válida si el planteamiento se realiza, no como medición de la productividad multifactorial a lo largo del tiempo en un solo país, sino como medición de los niveles comparativos de productividad multifactorial entre dos o más países en un momento dado, como lo hemos comprobado en otra investigación (Hernández Laos, 1985: 125-143). ${ }^{42}$

\footnotetext{
${ }^{42}$ Resulta interesante hacer notar que este resultado es similar al índice para la medición de niveles de productividad multifactorial entre países propuesto por Caves, Christensen y Diewert (1982), al que los autores denominan índice superlativo por basarse en números índices Törnqvist, los cuales son consistentes con una función de producción translogarítmica. El de ellos reclama, empero, de todos los supuestos ya conocidos sobre el comportamiento optimizador de los agentes y la existencia de rendimientos constantes a escala, en tanto que nuestra aproximación está exenta de todos esos supuestos.
} 


\section{BIBLIOGRAFÍA}

Abramovitz, M. 1956. "Resource and output trends in United States since 1870." American Economic Review 46 (2): 5-23.

Aigner, D. J., C. A. K. Lovell y P. Schmith. 1977. "Formulation and estimation of stochastic frontier production function models." Journal of Econometrics 6 (1): 21-37.

Atkinson, A. B. y J. E. Stiglitz. 1969. “A new view of technological change.” The Economic Journal 79 (315): 573-578.

Balk, B. M. 1993. "Malmquist productivity indexes and Fisher Ideal Indexes: Comment." The Economic Journal 103 (418): 680-682.

Balk, B. 1998. Industrial price, quantity and productivity indexes: The microeconomic theory and an application. Boston: Kluwer Academic Publishers.

Barton, G. T. y M. R. Cooper. 1948. "Relation of agricultural production to inputs." Review of Economics and Statistic 30(2): 117-26.

Barro, R. J. 1999. "Notes on growth accounting." Journal of Economic Growth 4: 119-137.

Barro, R. J. y X. Sala-i-Martin. 2005. Economic Growth Cambridge: The MIT Press. Bartelsman, E. J. y M. Doms. 2000. "Understanding productivity: Lessons from longitudinal microdata." Journal of Economic Literature 38 (3): 569-594.

Baily, M. N. y R. M. Solow. 2001. "International productivity comparisons built from the firm level." The Journal of Economic Perspectives 15 (3): 151-172.

Berndt, E. R. 1991. The practice of Econometrics: Classic and contemporary readings. Massachusetts: Addison-Wesley Publishing Co.

Carlaw, K. I. y R. G. Lipsey. 2003. "Productivity, technology and economic growth: What is the relationship?.” Journal of Economic Surveys 17 (3): 457-495.

Carlson, S. 1939. A study on the pure theory of production. London: King.

Caves, D. W., L. R. Christensen y W. E. Diewert. 1982. "Economic theory of index numbers and the measurement of input, output and productivity." Econometrica 50 (6): 1393-1414.

Christensen, L. R. y D. W. Jorgenson. 1969. "The measurement of U. S. real capital input, 1929-1967." Review of Income and Wealth 15(4): 293-320.

Coelli, T., D. S. Prasada Rao, y G. E. Battese. 1998. An introduction to efficiency and productivity analysis. Boston: Kluwer Academic Publishers.

Copeland, M. A. 1937. "Concepts of National Income." in Studies in income and wealth 1: 3-63. New York: National Bureau of Economic Research. 
Copeland, M. y E. M. Martin. 1938. "The correction of wealth and income estimates fro price changes." In Studies in income and wealth 2: 85-135. New York: National Bureau of Economic Research.

Debreu, G. 1951. "The coefficient of resource utilization." Econometrica 19 (3): 273-92.

Denison, E. F. 1967. Why growth rates differ?. Washington: Brookings Institution.

Diewert, W. E. 1976. "Exact and superlative index numbers." Journal of Econometrics 4: 115-45.

Diewert, W. E. 1978. "Superlative index numbers and consistency in aggregation." Econometrica 46: 883-900.

Diewert, W. E. 1982. "Duality approaches to microeconomic theory." In Handbook of Mathematical Economics edited by K. J. Arrow y M. D. Intriligator, Vol. II: 535-599. Amsterdam: North Hollad.

Diewert, W. E. 1983. "The measurement of waste within the production sector of an open economy." The Scandinavian Journal of Economics 85 (2): 159-179.

Diewert, W. E. 1992. "Fisher ideal output, input and productivity indexes revisited." Journal of Productivity Analysis 3: 211-248.

Diewert, E. W. y D. Lawrence. 1999. "Measuring New Zealand productivity." Treasury Working Paper 99/5, http://www.treasury.govt.nz/workingpapers/99-5.htm.

Diewert, E. W. y A. O. Nakamura. 2003. "Index number concepts, measures and decomposition of productivity growth." Journal of Productivity Analysis 19: $127-159$.

Färe, R. 1988. Fundamentals of production theory. In Lecture Notes, Economics and Mathematical Systems. Heidelberg: Springer-Verlag.

Färe, R., S. Grosskopf, y C. A. K. Lovell. 1985. The measurement of efficiency of production. Boston: Kluwer-Nijhoff.

Färe, R., S. Grosskopf, y C. A. K. Lovell. 1994a. Production frontiers. Cambridge: Cambridge University Press.

Fare, R., S. Grosskopf, M. Norris y Z. Zhang. 1994b. "Productivity growth, technical progress, and efficiency change in industrialized countries." American Economic Review 84 (1): 66-83.

Farrell, M. J. 1957. "The measurement of productive efficiency." Journal of the Royal Statistical Society, Series A, General, 120, Part 3: 253-81.

Fisher, I. 1927. The making of index numbers. Boston: Houghton Mifflin. 
Fisher, F. M. 1965. "Embodied technical change and the existence of an aggregate capital stock." Review of Economic Studies 32: 263-88.

Foray, D. 2004. The economics of knowledge. Cambridge: MIT Press.

Foster, L., J. Haltinwagner y C. J. Krizan. 2001. "Aggregate productivity growth from microeconomic evidence," en: Hulten, CH., E. R. Dean y D. M. Harper (Eds.), New developments in productivity analysis, Chicago: University of Chicago Press, 2001: 303-363.

Funker, H. y J. Voeller. 1978. "A note on the characterization of Fisher ideal index." en W. Eichhorn, R. Henn O. Opitz y R. W. Shephards (eds.), Theory and Aplications of Economic Indexes. Wurzburg: Physica-Verlag: 177-181.

Fuss, M. y D. McFaden (Eds.) 1978. Production economics: A dual approach to theory and applications. Amsterdam: North Holland.

Griliches, Z. 1994. "Productivity, R\&D, and the data constraint." American Economic Review 84 (1): 1-23.

Griliches, Z. 1996."The discovery of the residual: A historical note." Journal of Economic Literature 34 (3): 1324-1330.

Hall, R. E. 1988. "The relation between price and marginal cost in U. S. industry." Journal of Political Economy 96 (5): 921-947.

Harberger, A. C. 1998. "A vision of the growth process." American Economic Review 88 (1): 1-32

Helpman, E. (Ed.) 1998. General purpose technologies and economic growth. Cambridge: MIT Press.

Hernández Laos, E. 1973. Evolución de la productividad de los factores. México: Centro Nacional de Productividad.

Hernández Laos, E. 1985. La productividad y el desarrollo industrial en México. México: Fondo de Cultura Económica.

Hicks, J. R. 1940. "The valuation of the social income." Economica (N. S.) 7: 105-24.

Hicks, J. R. 1946. Value and capital. Segunda Edición. Oxford: Clarendon Press. Hilll, P. 2000. "Economic depreciation and the SNA." Paper presented at the $26^{\text {th }}$ Conference of International Association for Research on Income and Wealth, Cracovia, Polonia: Mimeo.

Hoff, K. y J. E. Stiglitz. 2001. "Modern economic theory and development." en G. E. Meier y J. E. Stiglitz (eds.) Frontiers of development economics. The future in perspective. The International Bank for Reconstruction and Development / The World Bank, Nueva York: Oxford University Press: $389-459$ 
Hsieh, Ch. 1998. "What explain the industrial revolution in East Asia?." Research Paper, University of California Berkeley. Enero.

Hulten, Ch. R. 1973. "Divisia index numbers." Econometrica 41: 1017-1025.

Hulten, Ch. R. 2001. "Total factor productivity: a short biography." En Hulten, Dean y Harper (eds.) New developments in productivity analysis. Chicago: University of Chicago Press.

Hulten, Ch. R., E. R. Dean y M. J. Harper (Eds.) 2001. New developments in productivity analysis. Chicago: University of Chicago Press.

Johnson, D. G. 1950. "The nature of the supply function for agricultural products." American Economic Review 40 (4): 539-64.

Jorgenson, D. W. y Z. Griliches. 1967. "The explanation of productivity change." Review of Economic Studies 34 (3): 249-83.

Jorgenson, D. 1995. Productivity Volume I: Poswar U. S. Economic Growth. Cambridge: MIT Press.

Kendrick, J. W. 1955. "Productivity" en S. Fabricant (ed), Government in economic life $35^{\text {th }}$ Annual Report. New York: NBER: 44-47

Kendrick, J. W. 1961. Productivity trends in the United States. Princeton: Princeton University Press.

Kumbhakar, S. C. y C. A. Lovell. 2000. Stochastic Frontier Analysis. Cambridge: Cambridge University Press.

Leibeinstein, H. 1966. “Allocative efficiency vs. X-efficiency.” American Economic Review 56 (3): 392-415.

Lydall, H. F. 1969. “On measuring technical progress.” Australian Economic Papers, 8 .

Nadiri, I. e I. Prucha. 2001. "Dynamic factor demand models and productivity analysis," en: Hulten, CH., E. R. Dean y D. M. Harper (Eds.), New developments in productivity analysis, Chicago: University of Chicago Press, 2001: 1-47.

Nataf, A. 1948. "Sur le possibilite de construction de certain macromodeles." Econometrica 16: 232-44.

OCDE. 2001. OECD Productivity Manual: A guide to the measurement of industry level and aggregate productivity growth. Paris: Organization for Economic Cooperation and Development. Marzo.

Richter, M. K. 1966. "Invariance axioms and economic indexes." Econometrica 37: 739-55.

Roscoe, E. S. 1963. Organization for production (3a edición). Homewood: Richard D. Irwin. 
Salter, W. E. G. 1966. Productivity and technical change (2a edición). Cambridge: Cambridge University Press.

Samuelson, P. A. 1947. Fundations of Economic Analysis. Cambridge: Harvard University Press.

Shreyer, P. y D. Pilat. 2001. "Measuring productivity." OECD Economic Studies 33: $127-170$.

Siegel, R. 1961. "On the design of consistent output and input indexes for productivity measurement." en NBER Output, input and productivity measurement. Princeton U. P: NBER.

Solow, R. M. 1957. "Technical change and the aggregate production function." Review of Economics and Statistics 39: 312-20.

Sschmookler, J. 1952. "The changing efficiency of the American Economy: 18691938." Review of Economics and Statistics 38 (3): 214-321

Stigler, G. J. 1947. Trends in output and employment. New York: NBER.

Tinbergen, J. 1942. "Zur theorie der langfirstigen wirtschsaftsentwicklung." Weltwirts Archiv, 1, Amsterdam, North Holland Publishing Co. pp. 522-49. Traducido al Ingles en: J. Tinmbergen, Selected Papers, North-Holland.

Tintner, G. 1944. "A note on the derivation of production functions from farm records." Econometrica 12 (1): 26-34.

Tybout, J. R. 2000. "Manufacturing firms in developing countries: How well do they do, and why?." Journal of Economic Literature 38 (1): 11-44. 